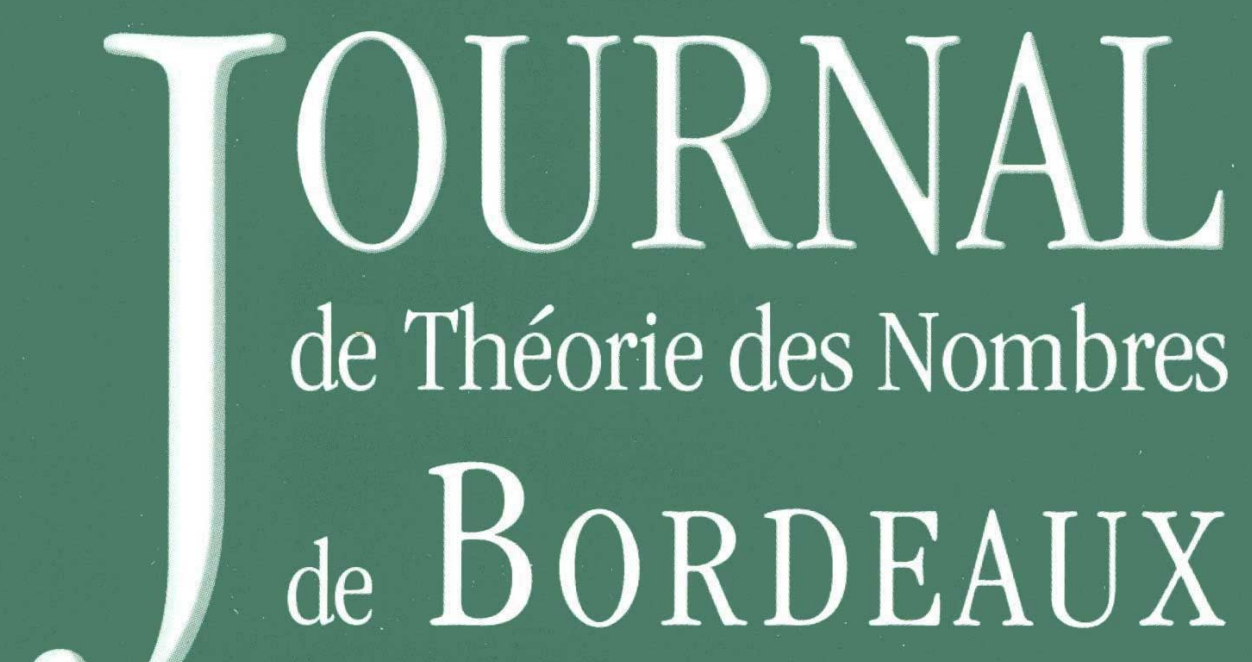

anciennement Séminaire de Théorie des Nombres de Bordeaux

Jörg M. THUSWALDNER

\title{
Unimodular Pisot substitutions and their associated tiles
}

Tome 18, no 2 (2006), p. 487-536.

<http://jtnb.cedram.org/item?id=JTNB_2006__18_2_487_0>

(C) Université Bordeaux 1, 2006, tous droits réservés.

L'accès aux articles de la revue «Journal de Théorie des Nombres de Bordeaux » (http://jtnb.cedram.org/), implique l'accord avec les conditions générales d'utilisation (http://jtnb.cedram. org/legal/). Toute reproduction en tout ou partie cet article sous quelque forme que ce soit pour tout usage autre que l'utilisation à fin strictement personnelle du copiste est constitutive d'une infraction pénale. Toute copie ou impression de ce fichier doit contenir la présente mention de copyright.

\section{cedram}




\title{
Unimodular Pisot substitutions and their associated tiles
}

\author{
par JÖRG M. THUSWALDNER
}

Dedicated to Professor Helmut Prodinger on the occasion of his $50^{\text {th }}$ birthday

RÉSUMÉ. Soit $\sigma$ une substitution de Pisot unimodulaire sur un alphabet à $d$ lettres et soient $X_{1}, \ldots, X_{d}$ les fractales de Rauzy associées. Dans le présent article, nous souhaitons étudier les frontières $\partial X_{i}(1 \leq i \leq d)$ de ces fractales. Dans ce but, nous définissons un graphe, appelé graphe de contact de $\sigma$ et noté $\mathcal{C}$. Si $\sigma$ satisfait une condition combinatoire appelée condition de super coïncidence, le graphe de contact peut être utilisé pour établir un système autoaffine dirigé par un graphe dont les attracteurs sont des morceaux des frontières $\partial X_{1}, \ldots, \partial X_{d}$. De ce système dirigé par un graphe, nous déduisons une formule simple pour la dimension fractale de $\partial X_{i}$, dans laquelle les valeurs propres de la matrice d'adjacence de $\mathcal{C}$ interviennent.

Un avantage du graphe de contact est sa structure relativement simple, ce qui rend possible sa construction immédiate pour une grande classe de substitutions. Dans cet article, nous construisons explicitement le graphe de contact pour une classe de substitutions de Pisot qui sont reliées aux $\beta$-développements par rapport à des unités Pisot cubiques. En particulier, nous considérons des substitutions de la forme

$$
\sigma(1)=\underbrace{1 \ldots 1}_{b \text { fois }} 2, \quad \sigma(2)=\underbrace{1 \ldots 1}_{a \text { fois }} 3, \quad \sigma(3)=1
$$

où $b \geq a \geq 1$. Il est bien connu que ces substitutions satisfont la condition de super coïncidence mentionnée plus haut. Donc nous pouvons donner une formule explicite pour la dimension fractale des frontìeres des fractales de Rauzy associées à ces substitutions.

Abstract. Let $\sigma$ be a unimodular Pisot substitution over a $d$ letter alphabet and let $X_{1}, \ldots, X_{d}$ be the associated Rauzy fractals. In the present paper we want to investigate the boundaries $\partial X_{i}(1 \leq i \leq d)$ of these fractals. To this matter we define a certain graph, the so-called contact graph $\mathcal{C}$ of $\sigma$. If $\sigma$ satisfies

Manuscrit reçu le 17 novembre 2004.

The author was supported by project S8310 of the Austrian Science Foundation. 
a combinatorial condition called the super coincidence condition the contact graph can be used to set up a self-affine graph directed system whose attractors are certain pieces of the boundaries $\partial X_{1}, \ldots, \partial X_{d}$. From this graph directed system we derive an easy formula for the fractal dimension of $\partial X_{i}$ in which eigenvalues of the adjacency matrix of $\mathcal{C}$ occur.

An advantage of the contact graph is its relatively simple structure, which makes it possible to construct it for large classes of substitutions at once. In the present paper we construct the contact graph explicitly for a class of unimodular Pisot substitutions related to $\beta$-expansions with respect to cubic Pisot units. In particular, we deal with substitutions of the form

$$
\sigma(1)=\underbrace{1 \ldots 1}_{b \text { times }} 2, \quad \sigma(2)=\underbrace{1 \ldots 1}_{a \text { times }} 3, \quad \sigma(3)=1
$$

where $b \geq a \geq 1$. It is well known that these substitutions satisfy the above mentioned super coincidence condition. Thus we can give an explicit formula for the fractal dimension of the boundaries of the Rauzy fractals related to these substitutions.

\section{Introduction}

In 1982 Rauzy [34] studied the Tribonacci substitution

$$
\sigma(1)=12, \quad \sigma(2)=13, \quad \sigma(3)=1 .
$$

He proved that the dynamical system generated by this substitution is measure-theoretically conjugate to an exchange of domains $X_{1}, X_{2}, X_{3}$ in a compact tile $X=X_{1} \cup X_{2} \cup X_{3}$. The set $X$ has fractal boundary. However, it is homeomorphic to a closed disk (cf. [30, Subsection 4.1]). Furthermore, the essentially disjoint basic tiles $X_{1}, X_{2}, X_{3}$ satisfy a self-similar graph directed system in the sense of Mauldin and Williams [28]. The set $X$ is now called the classical Rauzy fractal.

More generally, it is possible to attach a Rauzy fractal to each unimodular Pisot substitution. However, the structure of these fractals is more complicated in the general case. Arnoux and Ito [6] (compare also [4, 5, 11, 12, 40]) proved that the dynamical system associated to a unimodular Pisot substitution over a $d$ letter alphabet admits a conjugacy to an exchange of domains $X_{1}, \ldots, X_{d}$ in the compact set $X=X_{1} \cup \ldots \cup X_{d}$ provided that a certain combinatorial condition, the so-called strong coincidence condition is true. It is conjectured that this condition holds for each unimodular Pisot substitution. The topological structure of $X$ can be difficult. There exist substitutions over three letters whose Rauzy fractals are neither connected nor simply connected.

There are different ways to define the Rauzy fractal associated to a given unimodular Pisot substitution $\sigma$ over a $d$ letter alphabet. One possibility 
is via certain projections of a set of points related to a periodic point of $\sigma$ (see for instance [20, Chapter 8]). Another approach runs via so-called one dimensional "geometric realizations" of $\sigma$ and makes use of the duality principle of linear algebra (cf. [6]). Furthermore, Mosse [31] defined a certain "desubstitution map" on the dynamical system $\Omega$ associated to $\sigma$. With help of this map we can define a graph (the prefix-suffix automaton) that reveals a certain self-affinity property of $\Omega$. This reflects to a self-affinity property of the Rauzy fractal $X$ which allows to define $X$ by a graph directed system. This graph directed system is satisfied by the basic tiles $X_{1}, \ldots, X_{d}$ and is directed by the prefix-suffix automaton. All these definitions are equivalent and will be reviewed in Sections 2 and 3.

The fractal structure of the boundary of Rauzy fractals has been investigated firstly by Ito and Kimura [24]. They calculated the Hausdorff dimension of the boundary of the classical Rauzy fractal. Recently, Feng et al. [19] gave estimates for the Hausdorff dimension of Rauzy fractals associated to arbitrary unimodular Pisot substitutions. The approach used by Ito and Kimura in their dimension calculations makes use of two dimensional geometric realizations of the Tribonacci substitution. This construction has been considerably generalized and extended in Sano et al. [35] where a kind of Poincaré duality is established for higher dimensional geometric realizations of substitutions and their duals. Messaoudi [29, 30] studied geometric and topological properites of the classical Rauzy fractal.

Ito and Rao [25] investigate unimodular Pisot substitutions that admit the definition of tilings (see also Thurston [42]). In their paper they describe three different types of tilings which one can obtain using translates of basic tiles of Rauzy fractals. The existence of these tilings again depends on a combinatorial condition, the so-called super coincidence condition. Up to now it is not known whether this condition is satisfied for all unimodular Pisot substitutions or not. The tilings considered in [25], especially the aperiodic one, form the starting point of the present paper. We want to extend the notion of contact matrix (cf. [22]) and contact graph (cf. [37]) to the case of Rauzy fractals in order to study their boundaries. To this matter we use the above mentioned prefix-suffix automaton. In detail, this paper has the following aims.

In Section 2 we recall basic notations and give different equivalent definitions of the Rauzy fractal $X=X_{1} \cup \ldots \cup X_{d}$ associated to a given unimodular Pisot substitution $\sigma$ over a $d$ letter alphabet. Furthermore, we give relations between substitutions and a well-known notion of radix representations, the $\beta$-expansions. At the end of this section we define a tiling induced by translates of the basic tiles of a Rauzy fractal.

The main object of the present paper, the contact graph associated to a unimodular Pisot substitution, is defined in Section 3. We give a detailed 
discussion of the contact graph and illustrate it by some examples. In the setting of self-affine lattice tiles, the contact graph and its adjacency matrix (the contact matrix) have been used in order to derive tiling properties of these tiles and to investigate their boundaries (cf. [22, 37]).

From Section 4 onwards we assume that the substitutions under consideration satisfy the super coincidence condition (cf. Definition 4.1). We use the contact graph in order to represent the boundaries $\partial X$ and $\partial X_{i}$ of a Rauzy fractal $X=X_{1} \cup \ldots \cup X_{d}$ as a graph directed system (Theorem 4.3). In Feng et al. [19] as well as Siegel [39] other graph directed systems for the boundary of Rauzy fractals are given. However, it turns out that our construction is simpler than theirs and can be used to characterize the boundaries of whole classes of Rauzy fractals.

In Section 5 the above mentioned representation of $\partial X_{i}(1 \leq i \leq d)$ is used to derive an easy formula for the box counting dimension of $\partial X$ in which eigenvalues of the contact matrix occur (Theorem 5.9). In some cases we can even prove that the box counting dimension agrees with the Hausdorff dimension.

The main advantage of the contact graph is its relatively easy shape. In Section 6 we will calculate the contact graph for the substitutions

$$
\sigma(1)=\underbrace{1 \ldots 1}_{b \text { times }} 2, \quad \sigma(2)=\underbrace{1 \ldots 1}_{a \text { times }} 3, \quad \sigma(3)=1
$$

where $b \geq a \geq 1$. It turns out that it has roughly the same shape for each substitution from this class (cf. Theorem 6.4). The knowledge of the contact graphs of these substitutions enables us to establish an explicit formula for the Hausdorff dimension of the boundary of the associated Rauzy fractals (cf. Theorem 6.7).

The calculation of the fractal dimension of $\partial X$ and $\partial X_{i}$ is not the only possible application of the contact graph. In a forthcoming paper we will apply it in order to set up an algorithm which decides whether a given substitution satisfies the above mentioned super coincidence condition. Also the problem of addition in $\beta$-expansions seems to be related to the contact graph.

\section{Basic notions}

2.1. Substitutions. For $d \geq 2$ set $\mathcal{A}:=\{1, \ldots, d\}$. Let $\mathcal{A}^{*}$ be the set of all finite words on the alphabet $\mathcal{A}$ and let $\mathcal{A}^{\mathbb{Z}}$ be the set of doubly infinite sequences. As usual, $\mathcal{A}^{\mathbb{Z}}$ shall carry the product topology of the discrete topology on $\mathcal{A}$. The cylinder sets

$\left[u_{1} \cdot u_{2}\right]:=\left\{w=\left(\ldots w_{-1} \cdot w_{0} w_{1} \ldots\right): w_{-\left|u_{1}\right|} \ldots w_{-1} \cdot w_{0} w_{1} \ldots w_{\left|u_{2}\right|-1}=u_{1} \cdot u_{2}\right\}$

$\left(u_{1}, u_{2} \in \mathcal{A}^{*}\right)$ form a basis of this topology (if $u_{1}$ is empty the cylinder set is denoted just by $\left[u_{2}\right]$, if $u_{2}$ is empty we will write $\left.\left[u_{1} \cdot\right]\right)$. 
A substitution $\sigma$ is an endomorphism of the free monoid $\mathcal{A}^{*}$ which satisfies $\lim _{n \rightarrow \infty}\left|\sigma^{n}(i)\right|=\infty$ for at least one $i \in \mathcal{A}$. A substitution naturally extends to $\mathcal{A}^{\mathbb{Z}}$ by setting

$$
\sigma\left(\ldots w_{-2} w_{-1} \cdot w_{0} w_{1} w_{2} \ldots\right):=\ldots \sigma\left(w_{-2}\right) \sigma\left(w_{-1}\right) \cdot \sigma\left(w_{0}\right) \sigma\left(w_{1}\right) \sigma\left(w_{2}\right) \ldots
$$

The adjacency matrix of $\sigma$ is the $d \times d$ matrix defined by

$$
E_{0}(\sigma):=\left(a_{i j}\right)
$$

where $a_{i j}$ is the number of occurrences of the letter $i$ in $\sigma(j)$. If $E_{0}(\sigma)$ is a primitive matrix, we call $\sigma$ a primitive substitution.

Substitutions give rise to certain dynamical systems. Fundamental properties of these dynamical systems are surveyed in Queffélec [33]. Here we only need some simple facts about them. Let $\sigma$ be a substitution. We say that a doubly infinite sequence $w$ is a periodic point of $\sigma$ if there exists a positive integer $k$ with $\sigma^{k}(w)=w$. If we can choose $k=1$ then $w$ is called a fixed point of $\sigma$. In Queffélec [33] it is shown that each substitution has at least one periodic point. Let $\tau$ be the shift map on $\mathcal{A}^{\mathbb{Z}}$. It is defined by $\tau\left(\left(w_{i}\right)_{i \in \mathbb{Z}}\right)=\left(w_{i+1}\right)_{i \in \mathbb{Z}}$. A sequence $w \in \mathcal{A}^{\mathbb{Z}}$ with $\tau^{k}(w)=w$ is called $\tau$-periodic $(k \in \mathbb{N})$. The language $L(w)$ of $w \in \mathcal{A}^{\mathbb{Z}}$ is the set of all finite words occurring in $w$. Let $\Omega(w):=\left\{w^{\prime} \in \mathcal{A}^{\mathbb{Z}}: L\left(w^{\prime}\right) \subseteq L(w)\right\}$. Then the pair $(\Omega(w), \tau)$ is called the dynamical system generated by $w$.

Let $\sigma$ be primitive. Then $\Omega:=\Omega(w)$ is the same for each periodic point $w$ of $\sigma$. Thus we call $(\Omega, \tau)$ the dynamical system generated by $\sigma$.

Definition 2.1. Let $\sigma$ be a substitution with adjacency matrix $E_{0}(\sigma)$. If the characteristic polynomial of $E_{0}(\sigma)$ is the minimal polynomial of a Pisot number $\lambda$, we call $\sigma$ a Pisot substitution. If $\lambda$ is even a unit, we call $\sigma$ a unimodular Pisot substitution.

In the present paper we will frequently use two very well known examples of unimodular Pisot substitutions in order to illustrate our results. The Fibonacci substitution, which is defined by

$$
\sigma(1)=12, \quad \sigma(2)=1
$$

and the Tribonacci substitution

$$
\sigma(1)=12, \quad \sigma(2)=13, \quad \sigma(3)=1 .
$$

The matrix $E_{0}(\sigma)$ has the form

$$
E_{0}(\sigma)=\left(\begin{array}{ll}
1 & 1 \\
1 & 0
\end{array}\right) \quad \text { and } \quad E_{0}(\sigma)=\left(\begin{array}{lll}
1 & 1 & 1 \\
1 & 0 & 0 \\
0 & 1 & 0
\end{array}\right)
$$

for the Fibonacci and Tribonacci substitution, respectively.

It is easy to see that each Pisot substitution $\sigma$ is primitive (cf. for instance [12, Proposition 1.3]). In the present paper we are concerned with 
unimodular Pisot substitutions. For the case of Pisot substitutions which are not unimodular we refer to Berthé and Siegel [9] as well as Siegel [38], where some of their properties are discussed.

Holton and Zamboni [23] showed that each fixed point of a Pisot substitution $\sigma$ is not $\tau$-periodic. Thus the dynamical system $(\Omega, \tau)$ generated by $\sigma$ is infinite.

2.2. The prefix-suffix automaton. The shift space $\Omega$ of a primitive substitution is recognizable by a finite automaton. This automaton can be constructed with help of the desubstitution map $\theta$ which we will define now (cf. Mossé [31]). In [31] it is shown that each $w=\left(w_{\ell}\right)_{\ell \in \mathbb{Z}} \in \Omega$ admits a unique representation of the shape $w=\tau^{k} \sigma(y)$ with $y=\left(y_{\ell}\right)_{\ell \in \mathbb{Z}} \in \Omega$ and $0 \leq k<\left|\sigma\left(y_{0}\right)\right|$. Thus each $w$ may be written in the form $w=$ $\ldots \sigma\left(y_{-1}\right) \sigma\left(y_{0}\right) \sigma\left(y_{1}\right) \ldots$ with $\sigma\left(y_{0}\right)=w_{-k} \ldots w_{-1} . w_{0} \ldots w_{l}$. We use the notations

$$
\begin{aligned}
& p=w_{-k} \ldots w_{-1} \quad\left(\text { prefix of } \sigma\left(y_{0}\right)\right), \\
& s=w_{1} \ldots w_{l} \quad\left(\text { suffix of } \sigma\left(y_{0}\right)\right) .
\end{aligned}
$$

Note that $w$ is completely defined by $y$ and the decomposition of $\sigma\left(y_{0}\right)$. Let

$$
\mathcal{P}:=\left\{(p, i, s) \in \mathcal{A}^{*} \times \mathcal{A} \times \mathcal{A}^{*}: \text { there exists } j \in \mathcal{A} \text { such that } \sigma(j)=p i s\right\}
$$

be the set of all possible decompositions of $\sigma\left(y_{0}\right)$. According to the above construction define the desubstitution map $\theta$ and the partition map $\gamma$ by

$$
\begin{array}{ll}
\theta: \Omega \rightarrow \Omega, w \mapsto y & \text { such that } w=\tau^{k} \sigma(y) \text { and } 0 \leq k<\left|\sigma\left(y_{0}\right)\right|, \\
\gamma: \Omega \rightarrow \mathcal{P}, w \mapsto\left(p, w_{0}, s\right) & \text { such that } \sigma\left(y_{0}\right)=p w_{0} s \text { and } k=|p| .
\end{array}
$$

With help of these maps we define the prefix-suffix development of $w \in \Omega$ by the map

$$
G(w)=\left(\gamma\left(\theta^{\ell} w\right)\right)_{\ell \geq 0}=\left(p_{\ell}, i_{\ell}, s_{\ell}\right)_{\ell \geq 0} .
$$

Related to this map is the following automaton.

Definition 2.2. The prefix-suffix automaton $\Gamma_{\sigma}$ associated to a substitution $\sigma$ has

- Set of states $\mathcal{A}$. Each of the states is an initial state.

- Set of labels $\mathcal{P}$.

- There exists an edge from $i$ to $j$ labelled by $e=(p, i, s)$ if and only if $\sigma(j)=$ pis.

For a given substitution the prefix-suffix automaton can be constructed easily. For the Fibonacci and the Tribonacci substitution we get the graphs in Figure 1. 

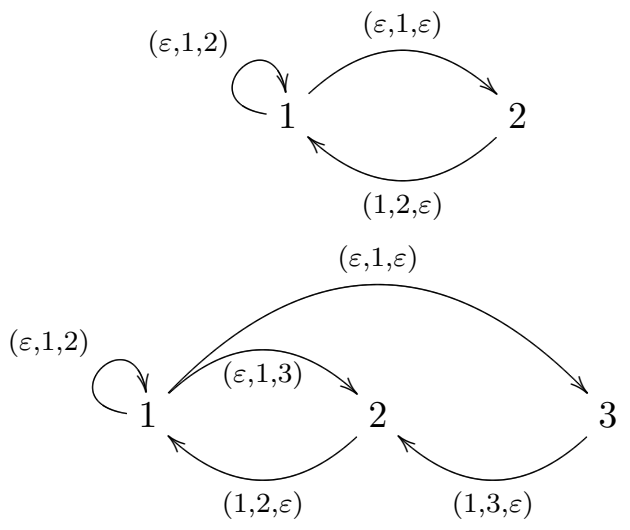

Figure 1. The prefix-suffix automata corresponding to the Fibonacci (above) and the Tribonacci substitution (below).

Let $\mathcal{D} \subset \mathcal{P}^{\mathbb{N}}$ be the set of labels of infinite walks in $\Gamma_{\sigma}$. According to [11] the map $G$ is continuous and maps onto $\mathcal{D}$. It is one-to-one except at the orbit of periodic points of $\sigma$. This implies that the sets

$$
\tau^{k} \sigma[i] \quad(i \in \mathcal{A}, k<|\sigma(i)|)
$$

partition $\Omega$ with countable overlap (cf. [11, Proposition 6.2]). For each $i \in \mathcal{A}$ we get the decomposition

$$
[i]=\bigcup_{\substack{j \in \mathcal{A},(p, i, s) \in \mathcal{P} \\ \sigma(j)=p i s}} \tau^{|p|} \sigma[j] .
$$

An analogous representation can be obtained for suffixes. Just note that for $\sigma(j)=$ pis we have $\tau^{|p|} \sigma[j]=\tau^{-|s|-1} \sigma[j$.$] . Thus (2.1) implies$

$$
[i .]=\bigcup_{\substack{j \in \mathcal{A},(p, i, s) \in \mathcal{P} \\ \sigma(j)=p i s}} \tau^{-|s|} \sigma[j .] .
$$

2.3. Construction of the Rauzy fractal. Our first aim is to define a tile $X$ related to a unimodular Pisot substitution $\sigma$. Such a tile was first defined for the Tribonacci substitution by Rauzy [34]. First we need the abelianization $f$ of $\sigma$. It is defined as follows. Let $e_{i}$ be the canonical $i$-th basis vector of $\mathbb{R}^{d}$. Then

$$
\begin{aligned}
f: \mathcal{A} & \rightarrow \mathbb{Z}^{d}, \\
i & \mapsto e_{i} .
\end{aligned}
$$


The domain of $f$ is extended to $\mathcal{A}^{*}$ in the following way. Let $w=$ $w_{1} \ldots w_{m} \in \mathcal{A}^{*}$. Then $f(w):=\sum_{\ell=1}^{m} f\left(w_{\ell}\right)$. It is easy to see that $f \circ \sigma=$ $E_{0}(\sigma) \circ f$.

Let $\lambda=\lambda_{1}>1, \lambda_{2}, \ldots, \lambda_{r}, \lambda_{r+1}, \bar{\lambda}_{r+1}, \ldots, \lambda_{r+s}, \bar{\lambda}_{r+s}(d=r+2 s)$ be the eigenvalues of $E_{0}(\sigma)$. and let

$$
U_{\mathbb{C}}:=\left\{u=u_{1}>0, u_{2}, \ldots, u_{r}, u_{r+1}, \bar{u}_{r+1}, \ldots, u_{r+s}, \bar{u}_{r+s}\right\}
$$

be a basis of $\mathbb{C}^{d}$ of right eigenvectors. Then we can select a basis

$$
V_{\mathbb{C}}:=\left\{v=v_{1} \geq 1, v_{2}, \ldots, v_{r}, v_{r+1}, \bar{v}_{r+1}, \ldots, v_{r+s}, \bar{v}_{r+s}\right\}
$$

of left eigenvectors such that $U_{\mathbb{C}}$ and $V_{\mathbb{C}}$ are dual bases, i.e.

$$
\begin{aligned}
\text { if } k=1, \ldots, r & \left\{\begin{array}{l}
u_{k} \cdot v_{k}=1, \\
u_{j} \cdot v_{k}=\bar{u}_{j} \cdot v_{k}=0 \quad \text { for } j \neq k,
\end{array}\right. \\
\text { if } k=r+1, \ldots, r+s & \left\{\begin{array}{l}
\bar{u}_{k} \cdot v_{k}=1, \\
u_{k} \cdot v_{k}=0, \\
u_{j} \cdot v_{k}=\bar{u}_{j} \cdot v_{k}=0
\end{array} \text { for } j \neq k .\right.
\end{aligned}
$$

In [12, Lemma 2.4] it is shown that

$$
\left\{u_{1}, u_{2}, \ldots, u_{r}, \Re u_{r+1}, \Im u_{r+1}, \ldots, \Re u_{r+s}, \Im u_{r+s}\right\}
$$

forms a basis of $\mathbb{R}^{d}$. This basis is used to define the contracting invariant hyperplane

$$
\mathbf{P}:=u_{2} \oplus \cdots \oplus u_{r} \oplus \Re u_{r+1} \oplus \Im u_{r+1} \oplus \cdots \oplus \Re u_{r+s} \oplus \Im u_{r+s}
$$

of $E_{0}(\sigma)$ (regarded as a linear operator on $\mathbb{R}^{d}$ ). Note that by the duality of $U_{\mathbb{C}}$ and $V_{\mathbb{C}}$ we have $\mathbb{R}^{d}=v \oplus \mathbf{P}$.

For $2 \leq k \leq r+s$ let $\delta_{k}: \mathcal{A}^{*} \rightarrow \mathbb{R}$ or $\mathbb{C}$ be the mapping which maps $w \in \mathcal{A}^{*}$ to the scalar product $f(w) \cdot v_{k}$. Furthermore, let

$$
\begin{aligned}
\delta: \mathcal{A}^{*} & \rightarrow \mathbb{R}^{r-1} \times \mathbb{C}^{s}, \\
w & \mapsto\left(\delta_{k}(w)\right)_{2 \leq k \leq r+s} .
\end{aligned}
$$

Obviously, $\delta$ is a homomorphism. With help of this map we define the representation map $\psi: \Omega \rightarrow \mathbb{R}^{r-1} \times \mathbb{C}^{s}$ by

$$
\psi(w)=\lim _{n \rightarrow \infty} \delta\left(\sigma^{0}\left(s_{0}\right) \ldots \sigma^{n}\left(s_{n}\right)\right)=\left(\begin{array}{c}
\sum_{\ell \geq 0} \delta_{2}\left(s_{\ell}\right) \lambda_{2}^{\ell} \\
\vdots \\
\sum_{\ell \geq 0} \delta_{r+s}\left(s_{\ell}\right) \lambda_{r+s}^{\ell}
\end{array}\right) .
$$

Here $\left(p_{\ell}, i_{\ell}, s_{\ell}\right)_{\ell \geq 0}$ denotes the prefix-suffix representation of $w \in \Omega$.

Let $\pi: \mathbb{R}^{d} \rightarrow \mathbf{P}$ be the linear projection of $\mathbb{R}^{d}$ to $\mathbf{P}$ along $u$. Since $U_{\mathbb{C}}$ and $V_{\mathbb{C}}$ are dual bases it is easy to see that $\pi$ can be written as $\pi(x)=x-(x \cdot v) u$. 
Let $\left.E_{0}(\sigma)\right|_{\mathbf{P}}$ be the restriction of the linear operator $E_{0}(\sigma)$ to the contractive hyperplane $\mathbf{P}$ and set

$$
\Sigma_{r+k}=\left(\begin{array}{cc}
\Re \lambda_{r+k} & \Im \lambda_{r+k} \\
-\Im \lambda_{r+k} & \Re \lambda_{r+k}
\end{array}\right) \quad(1 \leq k \leq s) .
$$

In what follows we identify $\mathbb{C}$ with $\mathbb{R}^{2}$ via the mapping $z \mapsto(\Re z, \Im z)$ and $\{0\} \times \mathbb{R}^{d-1}$ with $\mathbb{R}^{d-1}$ via the mapping $\left(0, z_{1}, \ldots, z_{d-1}\right) \mapsto\left(z_{1}, \ldots, z_{d-1}\right)$. Then there exists a regular real matrix $T_{\mathbf{P}}$ such that $\left.E_{0}(\sigma)\right|_{\mathbf{P}}=T_{\mathbf{P}}^{-1} \Lambda T_{\mathbf{P}}$ with

$$
\Lambda=\operatorname{diag}\left(\lambda_{2}, \ldots, \lambda_{r}, \Sigma_{r+1}, \ldots, \Sigma_{r+s}\right)
$$

and $\pi f=T_{\mathbf{P}}^{-1} \delta$ holds. Now we set

$$
\begin{aligned}
X_{i} & :=T_{\mathbf{P}}^{-1} \psi([i]) \quad(i \in \mathcal{A}), \\
X & :=\bigcup_{i \in \mathcal{A}} X_{i} .
\end{aligned}
$$

$X_{i}(i \in \mathcal{A})$ and $X$ are compact subsets of the contracting hyperplane $\mathbf{P}$. We call these sets the atomic surfaces or the Rauzy fractals of $\sigma$. In Figure 2 the atomic surfaces of the Fibonacci and Tribonacci substitution are depicted.
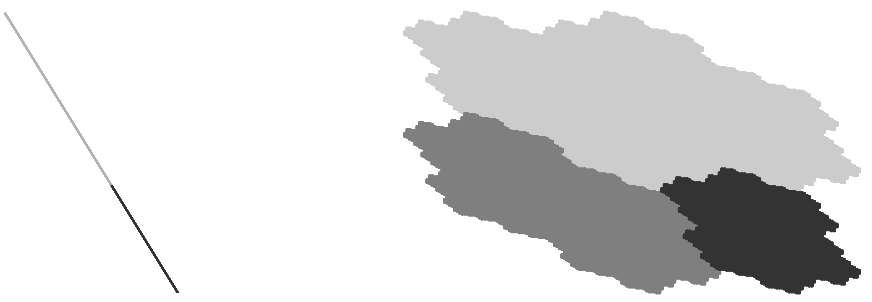

FiguRE 2. The atomic surfaces associated to the Fibonacci (left) and the Tribonacci (right) substitution.

The sets $X_{i}(i \in \mathcal{A})$ will form the prototiles in the tiling we are going to define. We mention also the following alternative definition of $X$ and $X_{i}$. Let $w=\left(w_{\ell}\right) \in \Omega$ be a periodic point of $\sigma$. Then

$$
\begin{aligned}
X_{i} & =-\overline{\left\{\pi f\left(w_{1} \ldots w_{k}\right): k \geq 0, w_{k}=i\right\}} \quad(i \in \mathcal{A}), \\
X & =-\overline{\left\{\pi f\left(w_{1} \ldots w_{k}\right): k \geq 0\right\}} .
\end{aligned}
$$

In Proposition 3.1 we will show that this definition agrees with the definition of $X_{i}$ and $X$ given above.

Another possibility to define the sets $X_{i}$ runs via graph directed systems. Before we give the details we recall the definition of a graph directed system. Let $G$ be a finite directed graph $G=G(\mathcal{V}, \mathcal{E})$ with the property that each of its states has an outgoing edge. Let $\mathcal{V}=\{1, \ldots, q\}$ be its set of states and 
$\mathcal{E}$ its set of edges. To each edge $\epsilon \in \mathcal{E}$ assign a contractive affine mapping $\xi_{\epsilon}$. We say that a set $A=A_{1} \cup \ldots \cup A_{q}$ is a graph directed self-affine set if

$$
A_{i}=\bigcup_{\epsilon: i \rightarrow j} \xi_{\epsilon}\left(A_{j}\right) \quad(1 \leq i \leq q)
$$

where the union is extended over all edges $\epsilon$ of $G$ starting at $i$. The relations (2.4) are called a graph directed system. If the mappings $\xi_{\epsilon}$ are similarities, we call $A=A_{1} \cup \ldots \cup A_{q}$ a graph directed self-similar set. The non-empty compact sets $A_{1}, \ldots, A_{q}$ are uniquely defined by the graph directed system in (2.4). This can be shown with help of a fix point argument. For a detailed account on graph directed sets we refer the reader to [28].

Observe that $\psi$ is one-to-one almost everywhere on each cylinder $[i$.$] (cf.$ [12, Proposition 4.4]). Now (2.2) yields the following self-affinity property of the sets $X_{i}$.

$$
X_{i}=\bigcup_{\substack{(p, i, s), j \\(p, i, s)}} \bigcup_{i} E_{0}(\sigma) X_{j}+\pi f(s) .
$$

Note that obviously $E_{0}(\sigma) \circ \pi=\pi \circ E_{0}(\sigma)$ and the non-overlapping union is extended over all edges in $\Gamma_{\sigma}$ starting at $i$. Since $E_{0}(\sigma)$ is a contraction on $\mathbf{P},(2.5)$ is a graph directed system and can be taken as the definition of $X_{i}(i \in \mathcal{A})$. Thus $X$ is a graph directed self-affine set.

For the Fibonacci substitution the set equation (2.5) reads

$$
X_{1}=\left(E_{0}(\sigma) X_{1}+\pi\left(\begin{array}{l}
0 \\
1
\end{array}\right)\right) \cup E_{0}(\sigma) X_{2}, \quad X_{2}=E_{0}(\sigma) X_{1},
$$

for the Tribonacci substitution we get the system

$$
\begin{aligned}
& X_{1}=\left(E_{0}(\sigma) X_{1}+\pi\left(\begin{array}{l}
0 \\
1 \\
0
\end{array}\right)\right) \cup\left(E_{0}(\sigma) X_{2}+\pi\left(\begin{array}{l}
0 \\
0 \\
1
\end{array}\right)\right) \cup E_{0}(\sigma) X_{3}, \\
& X_{2}=E_{0}(\sigma) X_{1} \\
& X_{3}=E_{0}(\sigma) X_{2} .
\end{aligned}
$$

It has been shown by Sirvent and Wang [40, Theorem 4.1] that $X_{i}$ has non-empty interior for each $i \in \mathcal{A}$. In fact, they even proved that $X_{i}=$ $\overline{\operatorname{int}\left(X_{i}\right)}$.

We want to sum up the results discussed previously in the following proposition.

Proposition 2.3. Let $\sigma$ be a unimodular Pisot substitution of d letters and let $X_{i}(i \in \mathcal{A})$ and $X=X_{1} \cup \ldots \cup X_{d}$ be the associated Rauzy fractals. Then $X$ and $X_{i}(i \in \mathcal{A})$ are non-empty compact sets that are uniquely determined 
by the graph directed system

$$
X_{i}=\bigcup_{\substack{(p, i, s), j \\(p, i, s)}}^{\longrightarrow} E_{0}(\sigma) X_{j}+\pi f(s) .
$$

Furthermore, $X$ and $X_{i}$ are regular sets in the sense that

$$
X=\overline{\operatorname{int}(X)} \quad \text { and } \quad X_{i}=\overline{\operatorname{int}\left(X_{i}\right)} \quad(i \in \mathcal{A}) \text {. }
$$

2.4. Relations to $\beta$-expansions. Atomic surfaces are strongly related to $\beta$-expansions of real numbers with respect to a Pisot unit. For a real number $\beta>1$ we define the $\beta$-transformation

$$
\begin{aligned}
T_{\beta}:[0,1] & \rightarrow[0,1), \\
x & \mapsto \beta x \bmod 1 .
\end{aligned}
$$

The $\beta$-expansion of $x \in[0,1]$ is defined by

$$
x=\sum_{\ell \geq 1} u_{\ell} \beta^{-\ell}
$$

where the "digits" $u_{\ell} \in\{0,1, \ldots,\lceil\beta\rceil-1\}$ are given by $u_{\ell}:=\left\lfloor\beta T_{\beta}^{\ell-1}(x)\right\rfloor$. Let $d_{\beta}(1)=u_{1} u_{2} \ldots$ denote the digit string corresponding to the $\beta$-expansion of 1 . The structure of $d_{\beta}(1)$ reflects many properties of the associated $\beta$-expansions (cf. [2]). If $\beta$ is a unimodular Pisot unit and the length of $d_{\beta}(1)$ is equal to the degree of $\beta$ we can find strong relations between $\beta$-expansions and prefix-suffix expansions. For instance, let $\operatorname{Irr}_{\beta}(x)=$ $x^{d}-k_{1} x^{d-1}-\cdots-k_{d-1} x-1$ with $k_{1} \geq k_{2} \geq \cdots \geq k_{d-1} \geq 1$ be the minimal polynomial of a Pisot unit $\beta$. In this case we have $d_{\beta}(1)=k_{1} k_{2} \ldots k_{d-1} 1$ (cf. [21, Theorem 2]) and we can associate to $\beta$ the substitution

$$
\sigma_{\beta}(j)=\underbrace{1 \ldots 1}_{k_{j} \text {-times }}(j+1) \quad(1 \leq j \leq d-1), \quad \sigma_{\beta}(d)=1
$$

(cf. $[10,26])$. The admissible $\beta$-expansions (cf. [32]) are exactly the expansions of the shape

$$
\sum_{\ell=0}^{\infty}\left|p_{\ell}\right| \beta^{-\ell}
$$

where $\left(p_{\ell}, i_{\ell}, s_{\ell}\right)_{\ell \geq 0}$ is the labelling of a reversed path in $\Gamma_{\sigma}$. The fundamental domains associated to these expansions (cf. for instance $[1,2,3]$ ) are the same as the atomic surfaces apart from affine transformations.

For $\operatorname{Irr}_{\beta}(x)=x^{2}-x-1$ we have $\beta=\frac{1+\sqrt{5}}{2}$. In this case the admissible $\beta$-expansions are characterized by the prefix-suffix automaton of the Fibonacci substitution depicted on the left hand side of Figure 1. From this graph it is easy to see that a digit sequence $\left\{u_{\ell}\right\}_{\ell \geq 1} \in\{0,1\}^{\mathbb{N}}$ gives rise to an admissible $\beta$-expansion (2.7) if and only if it does not contain the 
pattern 11. Similarly, one can check that the $\beta$-expansions with respect to the root of $x^{3}-x^{2}-x-1=0$ correspond to the Tribonacci substitution. Their admissible sequences must not contain the pattern 111 . The fundamental domains associated to these $\beta$-expansions are affine images of the atomic surfaces depicted in Figure 2.

Note that the above correspondence does not hold for $\beta$-expansions where the length of $d_{\beta}(1)$ is bigger than the degree of $\beta$ or even infinite (cf. [2]). In this case the corresponding substitutions need more letters (cf. [10]) and their geometric realizations do not fit in our framework.

2.5. Stepped surface and tilings. Since $\mathbf{P}=\left\{x \in \mathbb{R}^{d}: x \cdot v=0\right\}$ we set

$$
\begin{aligned}
& \mathbf{P}^{\geq 0}:=\left\{x \in \mathbb{R}^{d}: x \cdot v \geq 0\right\}, \\
& \mathbf{P}^{<0}:=\left\{x \in \mathbb{R}^{d}: x \cdot v<0\right\} .
\end{aligned}
$$

For $x \in \mathbb{Z}^{d}$ and $i \in \mathcal{A}$ let $[x, i]:=\left\{x-e_{i}+\theta e_{i}: \theta \in[0,1]\right\}$ be a line of length 1 in $\mathbb{R}^{d}$ and

$$
\left[x, i^{*}\right]:=\left\{x+\theta_{1} e_{1}+\cdots+\theta_{i-1} e_{i-1}+\theta_{i+1} e_{i+1}+\cdots+\theta_{d} e_{d}: \theta_{i} \in[0,1]\right\}
$$

a $(d-1)$-dimensional cube in $\mathbb{R}^{d}$ (note that in what follows we set $-\left[x, i^{*}\right]=$ $\left.\left[-x, i^{*}\right]\right)$. With help of this notion we define the stepped surface $S$

$$
S:=\left\{\left[x, i^{*}\right]: x \in \mathbb{Z}^{d}, 1 \leq i \leq d \text { such that } x \in \mathbf{P}^{\geq 0} \text { and } x-e_{i} \in \mathbf{P}^{<0}\right\} .
$$

Following [6] we call the elements of $S$ unit tips. The subset of $S$ with zero translates is especially needed in what follows. Thus we set $S_{0}:=\left\{\left[0, i^{*}\right]\right.$ : $i \in \mathcal{A}\} \subset S$. $S_{0}$ consists of three faces of the unit cube located at the origin.
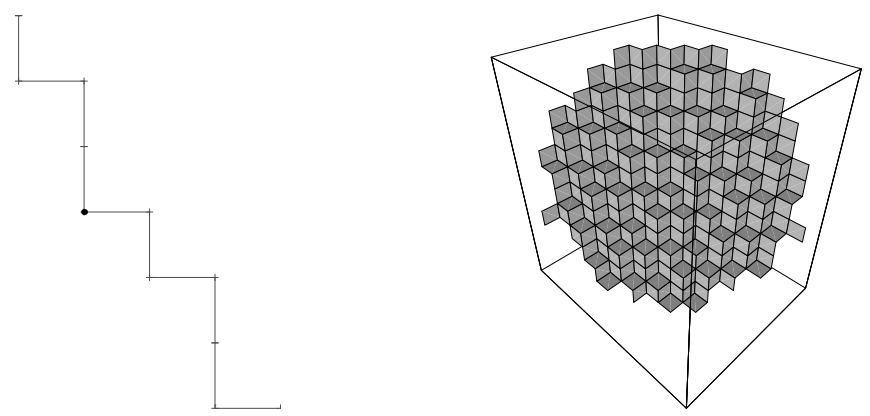

FiguRE 3. The stepped surfaces associated to the Fibonacci (left) and the Tribonacci (right) substitution.

In Figure 3 the stepped surfaces associated to the Fibonacci as well as the Tribonacci substitution are shown. 
Let $\sigma$ be an unimodular Pisot substitution and $\left\{X_{i}\right\}_{i \in \mathcal{A}}$ its atomic surfaces. It is conjectured that the collection

$$
\mathcal{I}:=\left\{\pi(x)+X_{i}:\left[x, i^{*}\right] \in S\right\}
$$

tiles the hyperplane $\mathbf{P}$ (cf. for instance [25]). Up to now, it has been shown that for unimodular Pisot substitutions this is equivalent to the so-called super coincidence condition (cf. [7, 25]; we will give the exact definition in Definition 4.1 below). This condition is conjectured to hold at least for each unimodular Pisot substitution. However, up to now it has been proved only for the case of Pisot substitutions with two letters (cf. Barge and Diamond [7]) as well as for some classes of substitutions with more letters. If this condition does not hold then overlaps occur.

\section{Definition of the contact graph}

3.1. Geometric realization of substitutions. With help of the prefixsuffix automaton we are in a position to define the one dimensional geometric realization $E_{1}(\sigma)$ of $\sigma$ (cf. [6] or [20, Chapter 8]).

$$
E_{1}(\sigma)[y, j]:=E_{0}(\sigma) y-\bigcup_{\substack{(p, i, s), i \\(p, i, s)}}^{\longrightarrow}[f(s), i] .
$$

The union is extended over all incoming edges of $j \in \mathcal{A}$ in the automaton $\Gamma_{\sigma}$. Note that $E_{1}^{n}(\sigma)[0, i](i \in \mathcal{A}, n \in \mathbb{N})$ is a broken line that approximates the direction of the eigenvector $u$. Furthermore, we need the dual of this map, namely

$$
E_{1}^{*}(\sigma)\left[x, i^{*}\right]:=E_{0}(\sigma)^{-1} x+\bigcup_{\substack{(p, i, s), j \\(p, i, s)}}^{\longrightarrow}\left[E_{0}(\sigma)^{-1} f(s), j^{*}\right] .
$$

Here the union is extended over all outgoing edges of $i \in \mathcal{A}$ in the automaton $\Gamma_{\sigma}$. Note that $E_{1}^{*}(\sigma)\left[x, i^{*}\right]$ is the union of all $\left[y, j^{*}\right]$ for which $[x, i]$ occurs in $E_{1}(\sigma)[y, j]$, i.e.

$$
\left[y, j^{*}\right] \in E_{1}^{*}(\sigma)\left[x, i^{*}\right] \quad \Longleftrightarrow \quad[x, i] \in E_{1}(\sigma)[y, j] .
$$

In the Fibonacci case we have

$$
\begin{aligned}
& E_{1}^{*}(\sigma)\left[x, 1^{*}\right]=E(\sigma)^{-1} x+\left(\left[e_{1}-e_{2}, 1^{*}\right] \cup\left[0,2^{*}\right]\right), \\
& E_{1}^{*}(\sigma)\left[x, 2^{*}\right]=E(\sigma)^{-1} x+\left[0,1^{*}\right] .
\end{aligned}
$$


In the Tribonacci case one computes

$$
\begin{aligned}
& E_{1}^{*}(\sigma)\left[0,1^{*}\right]=E(\sigma)^{-1} x+\left(\left[e_{1}-e_{3}, 1^{*}\right] \cup\left[e_{2}-e_{3}, 2^{*}\right] \cup\left[0,3^{*}\right]\right), \\
& E_{1}^{*}(\sigma)\left[0,2^{*}\right]=E(\sigma)^{-1} x+\left[0,1^{*}\right], \\
& E_{1}^{*}(\sigma)\left[0,3^{*}\right]=E(\sigma)^{-1} x+\left[0,2^{*}\right] .
\end{aligned}
$$

The dual of the one dimensional geometric realization of $\sigma$ can be used in order to approximate the atomic surfaces of $\sigma$. To make this clear, set

$$
\hat{X}_{i}(n):=\bigcup_{\left[y, j^{*}\right] \in E_{1}^{*}(\sigma)^{n}\left[0, i^{*}\right]} \pi\left[y, j^{*}\right]=\pi E_{1}^{*}(\sigma)^{n}\left[0, i^{*}\right] \quad(n \geq 0) .
$$

Note that $\hat{X}_{i}(0)=\pi\left[0, i^{*}\right]$. Let $n \geq 1$. Using the definition of $E_{1}^{*}(\sigma)$ we easily compute that

$$
\begin{aligned}
\hat{X}_{i}(n) & =\bigcup_{\left[y_{1}, j_{1}^{*}\right] \in E_{1}^{*}(\sigma)\left[0, i^{*}\right]\left[y, j^{*}\right] \in E_{1}^{*}(\sigma)^{n-1}\left[y_{1}, j_{1}^{*}\right]} \pi\left[y, j^{*}\right] \\
& =\bigcup_{\left[y_{1}, j_{1}^{*}\right] \in E_{1}^{*}(\sigma)\left[0, i^{*}\right]}\left(\hat{X}_{j_{1}}(n-1)+\pi E_{0}(\sigma)^{-(n-1)} y_{1}\right) \\
& =\bigcup_{i \stackrel{(p, i, s)}{\longrightarrow} j_{1}}\left(\hat{X}_{j_{1}}(n-1)+\pi E_{0}(\sigma)^{-n} f(s)\right) .
\end{aligned}
$$

Multiplying by $E_{0}(\sigma)^{n}$ and setting

$$
X_{i}(n)=E_{0}(\sigma)^{n} \hat{X}_{i}(n)
$$

we obtain

$$
X_{i}(n)=\bigcup_{i \stackrel{(p, i, s)}{\longrightarrow} j_{1}}\left(E_{0}(\sigma) X_{j_{1}}(n-1)+\pi f(s)\right) .
$$

This means that if we put $X_{j_{1}}(n-1)$ in the left hand side of the set equation (2.5) we obtain $X_{i}(n)$. By the general theory of graph directed systems (cf. for instance [18, Chapter 3] or [28]) this implies that

$$
\lim _{n \rightarrow \infty} X_{i}(n)=X_{i} \text { and } \lim _{n \rightarrow \infty} \bigcup_{i \in \mathcal{A}} X_{i}(n)=X
$$

in Hausdorff metric. Thus $E_{1}^{*}(\sigma)$ can be used to approximate the atomic surfaces in the sense of the Hausdorff metric.

In Figure 4 we see $E_{1}^{*}(\sigma)^{8}\left[0, i^{*}\right](i \in\{1,2\})$ for the Fibonacci as well as $E_{1}^{*}(\sigma)^{10}\left[0, i^{*}\right](i \in\{1,2,3\})$ for the Tribonacci substitution. In the first case, the approximation consists of unit line segments, in the second case it consists of unit squares.

For the sake of completeness we now sketch the proof of the following alternative representation of $X_{i}$ and $X$. 


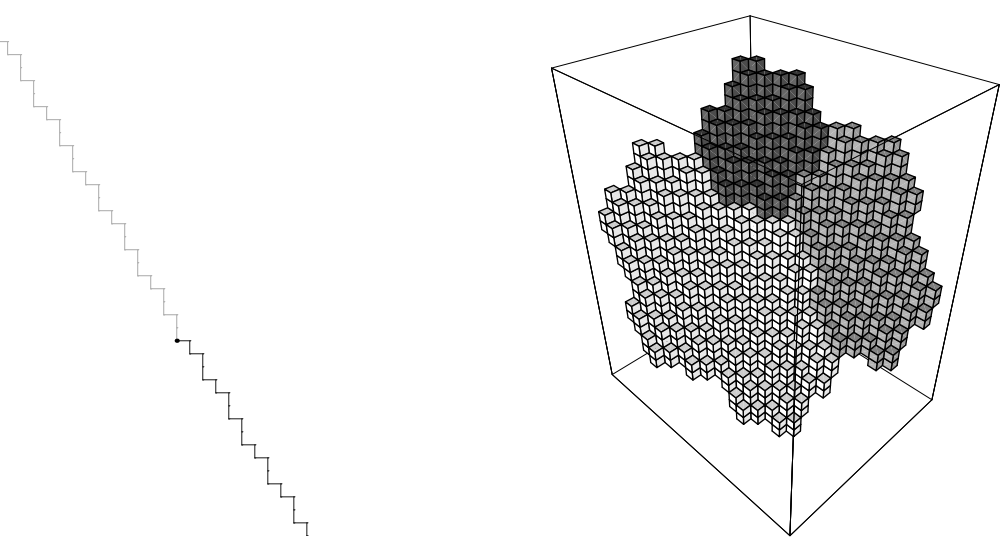

Figure 4. Approximation of the atomic surfaces with help of $E_{1}^{*}(\sigma)$ for the Fibonacci (left) and the Tribonacci (right) substitution.

Proposition 3.1. Let $w=\left(w_{\ell}\right)_{\ell \in \mathbb{N}} \in \Omega$ be a one sided periodic point of $\sigma$. Then

$$
\begin{aligned}
X_{i} & =-\overline{\left\{\pi f\left(w_{1} \ldots w_{k}\right): k \geq 0, w_{k}=i\right\}} \quad(i \in \mathcal{A}) \quad \text { and } \\
X & =-\overline{\left\{\pi f\left(w_{1} \ldots w_{k}\right): k \geq 0\right\} .}
\end{aligned}
$$

Proof. We prove the assertion only for $X$. For $X_{i}$ everything runs along similar lines. From the set equation (2.5) we see that changing $\sigma$ to $\sigma^{k}$ with $k \geq 1$ does not change the Rauzy fractals $X_{i}$ and $X$. Thus, since $\sigma$ is primitive, we may assume w.l.o.g. that $E_{0}(\sigma)$ is a positive matrix and that (after possible rearrangement of $\mathcal{A}$ ) $w=\left(w_{\ell}\right)_{\ell \in \mathbb{N}}=\lim _{n \rightarrow \infty} \sigma^{n}(1)$ is a one sided fix point of $\sigma$ starting with 1. By (3.4) we know that

$$
X=\lim _{n \rightarrow \infty} \pi \bigcup_{i \in \mathcal{A}} E_{0}(\sigma)^{n} E_{1}^{*}(\sigma)^{n}\left[0, i^{*}\right] .
$$

Using the duality between $E_{1}(\sigma)$ and $E_{1}^{*}(\sigma)$ we obtain

$$
X=-\lim _{n \rightarrow \infty} \pi \bigcup_{j \in \mathcal{A}} E_{1}(\sigma)^{n}[0, j] .
$$


Now we have

$$
\begin{aligned}
\lim _{n \rightarrow \infty} \pi E_{1}(\sigma)^{n}[0,1] & =\lim _{n \rightarrow \infty} \pi \underset{\substack{(p, i, s) \\
i(p, i, s)}}{\bigcup_{\longrightarrow}} E_{1}(\sigma)^{n-1}[-f(s), i] \\
& =\lim _{n \rightarrow \infty} \pi \bigcup_{\substack{(p, i, s) \\
i(p, i, s)}}^{\longrightarrow}\left(-E_{0}(\sigma)^{n-1} f(s)+E_{1}(\sigma)^{n-1}[0, i]\right) \\
& =\lim _{n \rightarrow \infty} \pi \bigcup_{j \in \mathcal{A}} E_{1}(\sigma)^{n-1}[0, j]
\end{aligned}
$$

where the last equality follows from the fact that $E_{0}(\sigma)$ is a positive matrix which is a contraction on $\mathbf{P}$. Thus

$$
X=-\lim _{n \rightarrow \infty} \pi E_{1}(\sigma)^{n}[0,1]
$$

This is still valid if we take only the limit of the vertices of the broken line $E_{1}(\sigma)^{n}[0,1]$. These vertices are exactly the points of the shape $f\left(w_{1} \ldots w_{k}\right)$ $(k \geq 0)$. Thus we may rewrite $(3.5)$ as

$$
X=-\overline{\left\{\pi f\left(w_{1} \ldots w_{k}\right): k \geq 0\right\}}
$$

This proves the result.

3.2. A sequence of sets related to the contact graph. Now we are in a position to construct certain sets, which lead to a generalization of the contact graph, which is well known for lattice tilings (cf. for instance $[22,37])$, to atomic surfaces. We will use the above mentioned approximation property in order to approximate the boundary of the sets $X_{i}$ by the boundary of the sets $X_{i}(n)$ defined in (3.3). We start with the description of $\partial X_{i}(0)$.

We easily see that

$$
\mathcal{I}_{0}:=\left\{\pi(x)+\pi\left[0, i^{*}\right]:\left[x, i^{*}\right] \in S\right\}
$$

is a tiling of $\mathbf{P}$ in the sense that it covers $\mathbf{P}$ with overlaps of zero measure. In [25, Theorem 2.5] (see also Arnoux-Ito [6]) it is shown that $E_{1}^{*}(\sigma)$ is a so-called "tiling-substitution" which means that

$$
\mathcal{I}_{n}:=\left\{\pi(x)+X_{i}(n):\left[x, i^{*}\right] \in S\right\}
$$

also forms a tiling of $\mathbf{P}$ for each $n \in \mathbb{N}$. First consider $\mathcal{I}_{0}$. Because $\mathcal{I}_{0}$ is a tiling of $\mathbf{P}$, the boundary of a tile $\pi\left[0, i^{*}\right](i \in \mathcal{A})$ is a union of sets of the form

$$
\pi\left(\left[0, i^{*}\right] \cap\left[y, j^{*}\right]\right) \quad\left(\pi\left[y, j^{*}\right] \in \mathcal{I}_{0},\left[y, j^{*}\right] \neq\left[0, i^{*}\right]\right) .
$$

Obviously, this union can be made finite because

$$
\left\{\pi(x):\left[x, i^{*}\right] \in S\right\}
$$


is a uniformly discrete subset of $\mathbf{P}$. Moreover, since the tiles are $(d-1)$ dimensional prisms, we only have to take intersections with tiles which pair a $(d-2)$-dimensional face with $\pi\left[0, i^{*}\right]$. Let $U_{i}$ be the set of all unit tips, which pair at least $(d-2)$-dimensional faces with the unit tip $\left[0, i^{*}\right](i \in \mathcal{A})$, i.e.

$$
U_{i}:=\left\{\left[y, j^{*}\right] \in S: \mathcal{L}_{d-2}\left(\left[y, j^{*}\right] \cap\left[0, i^{*}\right]\right)>0\right\}
$$

$\left(\mathcal{L}_{k}\right.$ is the $k$-dimensional Lebesgue measure). In Figure 5 we depict the elements of $U_{1} \cup U_{2}$ for the Fibonacci as well as the elements of $U_{1} \cup U_{2} \cup U_{3}$ for the Tribonacci substitution. In the case of the Fibonacci substitution the boundary of $\pi\left[0, i^{*}\right](i \in\{1,2\})$ consists of exactly two points (the end points of the line segment $\left.\left[0, i^{*}\right]\right)$. In the Tribonacci case the boundaries of the "central" unit tips $\left[0, i^{*}\right](i \in\{1,2,3\})$ are indicated by boldface lines. These boundaries are unions of line segments which come from the intersection of two unit tips.
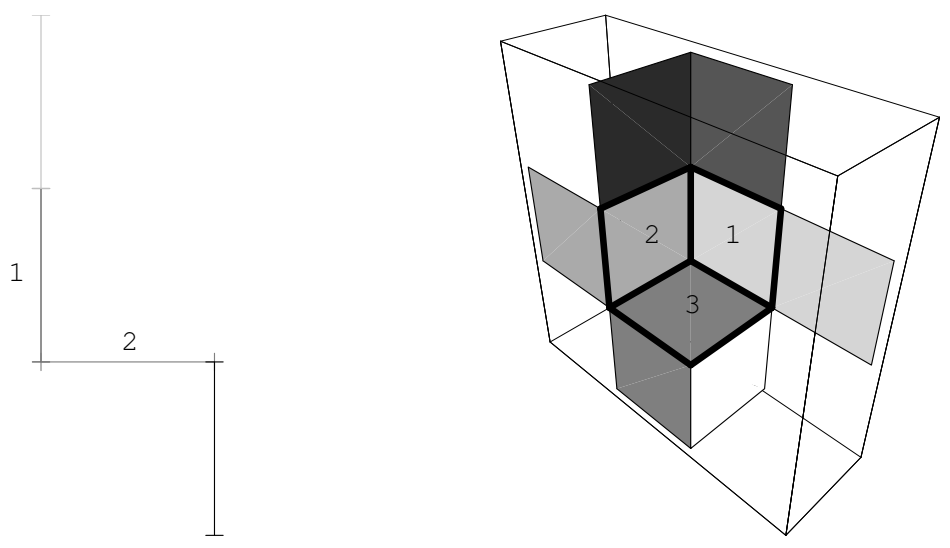

Figure 5. The unit tips which pair at least $(d-2)$ dimensional faces with the "central" unit tips $\left[0, i^{*}\right]$ for the Fibonacci (left) and the Tribonacci (right) substitution. The set $\left[0, i^{*}\right]$ is indicated by $i$.

Let

$$
\mathcal{Q}:=\left\{\left(\left[x, i^{*}\right],\left[y, j^{*}\right]\right):\left[x, i^{*}\right],\left[y, j^{*}\right] \in S \text { with } x=0 \text { or } y=0\right\} .
$$

Throughout the present paper we identify the elements $\left(\left[x, i^{*}\right],\left[y, j^{*}\right]\right)$ and $\left(\left[y, j^{*}\right],\left[x, i^{*}\right]\right)$. We will say that an element of $\mathcal{Q}$ is written in canonical order if it is written in the form

$$
\begin{cases}\left(\left[0, i_{1}^{*}\right],\left[z, i_{2}^{*}\right]\right) & \text { if } z \neq 0 \\ \left(\left[0, i_{1}^{*}\right],\left[0, i_{2}^{*}\right]\right) \text { with } i_{1} \leq i_{2} & \text { if } z=0\end{cases}
$$


In order to keep the analogy to the contact matrix of lattice tilings (cf. [22]) we set

$$
R_{0}:=\bigcup_{i \in \mathcal{A}}\left\{\left(\left[0, i^{*}\right],\left[y, j^{*}\right]\right) \in \mathcal{Q}:\left[y, j^{*}\right] \in U_{i}\right\}
$$

Note that $R_{0}$ is the set of all pairs which correspond to non-empty intersections of the form $\pi\left(\left[0, i^{*}\right] \cap\left[y, j^{*}\right]\right)$. Thus

$$
\partial \pi\left[0, i^{*}\right]=\bigcup_{\substack{\left(\left[0, i^{*}\right]\left[y, j^{*}\right]\right) \in R_{0} \\\left[0, i^{*}\right] \neq\left[y, j^{*}\right]}} \pi\left(\left[0, i^{*}\right] \cap\left[y, j^{*}\right]\right) \quad(i \in \mathcal{A}) .
$$

Looking at Figure 5 again, we see that for the Fibonacci substitution we have $\# R_{0}=5$ (two line segments plus three end points), while in the Tribonacci case we have $\# R_{0}=12$ (3 surfaces corresponding to the elements $\left(\left[0, i^{*}\right],\left[0, i^{*}\right]\right)$; furthermore, the boundary consists of 9 different line segments).

Thus $R_{0}$ contains full information on the boundary of the sets $X_{i}(0)=$ $\pi\left[0, i^{*}\right]$. We now want to set up a sequence of sets $R_{n}$ which contains full information on the boundaries of the sets $X_{i}(n)$. Before we do this in full generality, we want to provide the construction of $R_{1}$ starting from $R_{0}$ in full detail. To this end we define $\varphi: S \times S \rightarrow \mathcal{Q}$ by

$$
\varphi\left(\left[x_{1}, i_{1}^{*}\right],\left[x_{2}, i_{2}^{*}\right]\right):= \begin{cases}\left(\left[0, i_{1}^{*}\right],\left[x_{2}-x_{1}, i_{2}^{*}\right]\right) & \text { if }\left(x_{1}-x_{2}\right) \cdot v<0 \\ \left(\left[0, i_{2}^{*}\right],\left[x_{1}-x_{2}, i_{1}^{*}\right]\right) & \text { if }\left(x_{1}-x_{2}\right) \cdot v \geq 0\end{cases}
$$

It remains to check that $\varphi$ maps to $\mathcal{Q}$. If the first alternative in the definition of $\varphi$ holds it suffices to show that $\left[x_{2}-x_{1}, i_{2}^{*}\right] \in S$. For this alternative $\left(x_{2}-x_{1}\right) \cdot v>0$ holds. Furthermore, we have

$$
\left(x_{2}-x_{1}-e_{i_{2}}\right) \cdot v=\left(x_{2}-e_{i_{2}}\right) \cdot v-x_{1} \cdot v<-x_{1} \cdot v \leq 0
$$

because $\left[x_{1}, i_{1}^{*}\right],\left[x_{2}, i_{2}^{*}\right] \in S$. This proves that $\left[x_{2}-x_{1}, i_{2}^{*}\right] \in S$. The second alternative is treated likewise.

We set

$$
\begin{aligned}
R_{1}:=R_{0} \cup\left\{\varphi\left(\left[x_{1}, i_{1}^{*}\right],\left[x_{2}, i_{2}^{*}\right]\right) \in \mathcal{Q}: \exists\left(\left[0, j_{1}^{*}\right],\left[y, j_{2}^{*}\right]\right) \in R_{0}\right. \\
\left.\quad \text { with }\left[x_{1}, i_{1}\right] \in E_{1}(\sigma)\left[0, j_{1}\right] \text { and }\left[x_{2}, i_{2}\right] \in E_{1}(\sigma)\left[y, j_{2}\right]\right\} .
\end{aligned}
$$

Lemma 3.2. Let $\left[x_{1}, i_{1}^{*}\right]$ and $\left[x_{2}, i_{2}^{*}\right]$ be elements of $S$. Then $\pi\left(\left[x_{1}, i_{1}^{*}\right] \cap\right.$ $\left.\left[x_{2}, i_{2}^{*}\right]\right)$ forms a $(d-2)$-dimensional face if and only if $\varphi\left(\left[x_{1}, i_{1}^{*}\right],\left[x_{2}, i_{2}^{*}\right]\right) \in$ $R_{0}$.

Proof. This is an easy consequence of the definitions of $\varphi$ and $R_{0}$.

With these preparations we are able to prove the following result. 
Proposition 3.3. The boundary of $X_{i}(1)$ is given by

$$
\partial X_{i}(1)=\bigcup_{\substack{\left(\left[0, i^{*}\right]\left[y, j^{*}\right]\right) \in R_{1} \\\left[0, i^{*}\right] \neq\left[y, j^{*}\right]}}\left(X_{i}(1) \cap\left(X_{j}(1)+\pi(y)\right)\right) \quad(i \in \mathcal{A}) .
$$

Proof. As mentioned above, $\mathcal{I}_{1}$ forms a tiling of $\mathbf{P}$. Thus the boundary of the set $X_{i_{1}}(1)\left(i_{1} \in \mathcal{A}\right)$ consists of sets of the shape $X_{i_{1}}(1) \cap\left(X_{i_{2}}(1)+\pi(x)\right)$. Since $X_{i_{1}}(1)$ is a finite union of $(d-1)$-dimensional prisms, it suffices to take into account only intersections that contain at least one $(d-2)$-dimensional face. Because

$$
X_{i_{1}}(1)=\bigcup_{\left[y, j^{*}\right] \in E_{1}^{*}(\sigma)\left[0, i^{*}\right]} E_{0}(\sigma)\left(X_{j}(0)+\pi(y)\right)
$$

the intersection $X_{i_{1}}(1) \cap\left(X_{i_{2}}(1)+\pi(x)\right)$ contains a $(d-2)$-dimensional face if and only if there exist

$$
\left[y_{1}, j_{1}^{*}\right] \in E_{1}^{*}(\sigma)\left[0, i_{1}^{*}\right] \quad \text { and } \quad\left[y_{2}, j_{2}^{*}\right] \in E_{1}^{*}(\sigma)\left[x, i_{2}^{*}\right]
$$

such that $\pi\left(\left[y_{1}, j_{1}^{*}\right] \cap\left[y_{2}, j_{2}^{*}\right]\right)$ forms a $(d-2)$-dimensional face, i.e., in view of Lemma 3.2, if and only if

$$
\left[y_{1}, j_{1}^{*}\right] \in E_{1}^{*}(\sigma)\left[0, i_{1}^{*}\right],\left[y_{2}, j_{2}^{*}\right] \in E_{1}^{*}(\sigma)\left[x, i_{2}^{*}\right] \text { and } \varphi\left(\left[y_{1}, j_{1}^{*}\right],\left[y_{2}, j_{2}^{*}\right]\right) \in R_{0} \text {. }
$$

Using the duality relation (3.1) between $E_{1}(\sigma)$ and $E_{1}^{*}(\sigma)$ we see that (3.11) is equivalent to the existence of $\left[y_{1}, j_{1}^{*}\right],\left[y_{2}, j_{2}^{*}\right] \in S$ such that

$$
\left[0, i_{1}\right] \in E_{1}(\sigma)\left[y_{1}, j_{1}\right],\left[x, i_{2}\right] \in E_{1}(\sigma)\left[y_{2}, j_{2}\right] \text { and } \varphi\left(\left[y_{1}, j_{1}^{*}\right],\left[y_{2}, j_{2}^{*}\right]\right) \in R_{0} \text {. }
$$

We have to distinguish two cases according to the two cases in the definition of $\varphi$ in (3.9).

- Suppose first that $\varphi\left(\left[y_{1}, j_{1}^{*}\right],\left[y_{2}, j_{2}^{*}\right]\right)=\left(\left[0, j_{1}^{*}\right],\left[y_{2}-y_{1}, j_{2}^{*}\right]\right)$ holds and set $y=y_{2}-y_{1}$. Translating the first two statements of (3.12) by the vector $-E_{0}(\sigma) y_{1}$ yields

$\left[-E_{0}(\sigma) y_{1}, i_{1}\right] \in E_{1}(\sigma)\left[0, j_{1}\right],\left[x-E_{0}(\sigma) y_{1}, i_{2}\right] \in E_{1}(\sigma)\left[y, j_{2}\right]$

and

$$
\left(\left[0, j_{1}^{*}\right],\left[y, j_{2}^{*}\right]\right) \in R_{0} .
$$

Because $\left[x, i_{2}^{*}\right]$ is a unit tip we have

$$
\varphi\left(\left[-E_{0}(\sigma) y_{1}, i_{1}^{*}\right],\left[x-E_{0}(\sigma) y_{1}, i_{2}^{*}\right]\right)=\left(\left[0, i_{1}^{*}\right],\left[x, i_{2}^{*}\right]\right) .
$$

- Now suppose that $\varphi\left(\left[y_{1}, j_{1}^{*}\right],\left[y_{2}, j_{2}^{*}\right]\right)=\left(\left[0, j_{2}^{*}\right],\left[y_{1}-y_{2}, j_{1}^{*}\right]\right)$ holds and set $y=y_{1}-y_{2}$. Translating the first two statements of (3.12) by the vector $-E_{0}(\sigma) y_{2}$ yields

$\left[-E_{0}(\sigma) y_{2}, i_{1}\right] \in E_{1}(\sigma)\left[y, j_{1}\right],\left[x-E_{0}(\sigma) y_{2}, i_{2}\right] \in E_{1}(\sigma)\left[0, j_{2}\right]$ 
and

$$
\left(\left[0, j_{2}^{*}\right],\left[y, j_{1}^{*}\right]\right) \in R_{0} .
$$

Because $\left[x, i_{2}^{*}\right]$ is a unit tip we have

$$
\varphi\left(\left[-E_{0}(\sigma) y_{2}, i_{1}^{*}\right],\left[x-E_{0}(\sigma) y_{2}, i_{2}^{*}\right]\right)=\left(\left[0, i_{1}^{*}\right],\left[x, i_{2}^{*}\right]\right) .
$$

In both cases $(3.12)$ implies the existence of $\left[x_{1}, \tilde{i}_{1}^{*}\right],\left[x_{2}, \tilde{i}_{2}^{*}\right] \in \mathbb{Z}^{d} \times \mathcal{A}$ and $\left(\left[0, j_{1}^{*}\right],\left[y, j_{2}^{*}\right]\right) \in R_{0}$ such that

$$
\begin{gathered}
\left(\left[0, i_{1}^{*}\right],\left[x, i_{2}^{*}\right]\right)=\varphi\left(\left[x_{1}, \tilde{i}_{1}^{*}\right],\left[x_{2}, \tilde{i}_{2}^{*}\right]\right), \\
{\left[x_{1}, \tilde{i}_{1}\right] \in E_{1}(\sigma)\left[0, j_{1}\right] \text { and }\left[x_{2}, \tilde{i}_{2}\right] \in E_{1}(\sigma)\left[y, j_{2}\right]}
\end{gathered}
$$

By the definition of $R_{1}$ this implies that $\left(\left[0, i_{1}^{*}\right],\left[x, i_{2}^{*}\right]\right) \in R_{1}$.

Summing up we have shown that $X_{i_{1}}(1) \cap\left(X_{i_{2}}(1)+\pi(x)\right)$ contains a $(d-2)$-dimensional face only if $\left(\left[0, i_{1}^{*}\right],\left[x, i_{2}^{*}\right]\right) \in R_{1}$. Hence, we may write the boundary of $X_{i}(1)$ as

$$
\partial X_{i}(1)=\bigcup_{\substack{\left(\left[0, i_{1}^{*}\right]\left[x, i_{2}^{*}\right]\right) \in R_{1} \\\left[0, i_{1}^{*}\right] \neq\left[x, i_{2}^{*}\right]}}\left(X_{i_{i}}(1) \cap\left(X_{i_{2}}(1)+\pi(x)\right)\right) \quad(i \in \mathcal{A})
$$

and the result is proved.

The fact that we add $R_{0}$ in the definition of $R_{1}$ has technical reasons. It ensures that the sequence $\left\{R_{n}\right\}_{n \geq 0}$ defined below is increasing.

In Figure 6 the sets $E_{1}^{*}(\sigma)\left[y, j^{*}\right]$ for which $\left(\left[0, i^{*}\right]\left[y, j^{*}\right]\right) \in R_{1}$ are depicted. In the Fibonacci case we have

$$
\begin{aligned}
\hat{X}_{1}(1) & =\pi E_{1}^{*}(\sigma)\left[0,1^{*}\right]=\pi\left(\left[e_{1}-e_{2}, 1^{*}\right] \cup\left[0,2^{*}\right]\right), \\
\hat{X}_{2}(1) & =\pi E_{1}^{*}(\sigma)\left[0,2^{*}\right]=\pi\left[0,1^{*}\right] .
\end{aligned}
$$

In the Tribonacci case one computes

$$
\begin{aligned}
\hat{X}_{1}(1) & =\pi E_{1}^{*}(\sigma)\left[0,1^{*}\right]=\pi\left(\left[e_{1}-e_{3}, 1^{*}\right] \cup\left[e_{2}-e_{3}, 2^{*}\right] \cup\left[0,3^{*}\right]\right), \\
\hat{X}_{2}(1) & =\pi E_{1}^{*}(\sigma)\left[0,2^{*}\right]=\pi\left[0,1^{*}\right], \\
\hat{X}_{3}(1) & =\pi E_{1}^{*}(\sigma)\left[0,3^{*}\right]=\pi\left[0,2^{*}\right] .
\end{aligned}
$$

Each tile $E_{1}^{*}(\sigma)\left[y, j^{*}\right]$ is depicted in a different color. Observe that the tiles all have the property to pair at least one $(d-2)$-dimensional face with at least one of the "central" tiles $E_{1}^{*}(\sigma)\left[0, i^{*}\right]$. In the case of the Tribonacci substitution the boundaries of the sets $E_{1}^{*}(\sigma)\left[0, i^{*}\right]$ are indicated by bold face lines. 

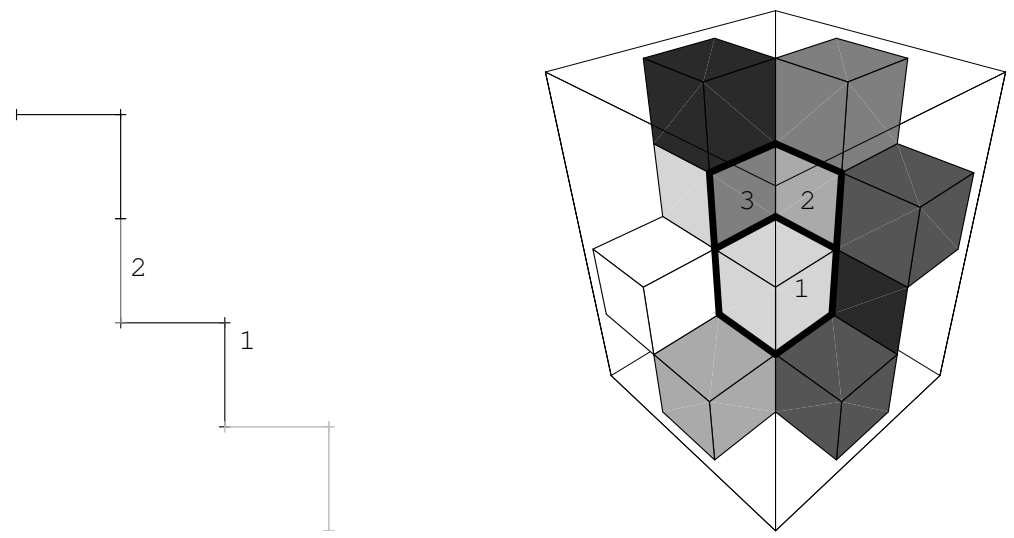

FiguRE 6. Illustration of the set $R_{1}$ for the Fibonacci (left) and the Tribonacci (right) substitution. The set $E_{1}^{*}(\sigma)\left[0, i^{*}\right]$ is indicated by $i$.

Now we turn to the general case. We construct $R_{n+1}$ starting from $R_{n}$ in the same way as we constructed $R_{1}$ starting from $R_{0}$. Thus starting with $R_{0}$ we define the sequence $\left\{R_{n}\right\}_{n \in \mathbb{N}}$ by a recurrence relation. This is done by the following set function. Let $2^{\mathcal{Q}}$ be the set of all subsets of $\mathcal{Q}$. Then

$$
\begin{aligned}
\Psi: 2^{\mathcal{Q}} & \rightarrow 2^{\mathcal{Q}} \\
M & \mapsto M \cup\left\{\varphi\left(\left[x_{1}, i_{1}^{*}\right],\left[x_{2}, i_{2}^{*}\right]\right) \in \mathcal{Q}: \exists\left(\left[0, j_{1}^{*}\right],\left[y, j_{2}^{*}\right]\right) \in M\right. \\
& \left.\quad \text { with }\left[x_{1}, i_{1}\right] \in E_{1}(\sigma)\left[0, j_{1}\right] \text { and }\left[x_{2}, i_{2}\right] \in E_{1}(\sigma)\left[y, j_{2}\right]\right\} .
\end{aligned}
$$

Now suppose that $R_{0}, \ldots, R_{n}$ are already defined. Then $R_{n+1}$ is defined in terms of $R_{n}$ by

$$
R_{n+1}:=\Psi\left(R_{n}\right) .
$$

In Lemma 4.2 we will show that the sets $R_{n}$ admit a generalization of (3.10) for the boundaries $\partial X_{i}(n)(n \geq 1)$. The following lemma shows that the sequence $\left\{R_{n}\right\}_{n \in \mathbb{N}}$ is ultimately constant.

Lemma 3.4. Let $\sigma$ be a unimodular Pisot substitution and attach to it the sequence of sets $\left\{R_{n}\right\}$ defined in (3.13). Then there exists an effectively computable integer $N \in \mathbb{N}$ such that $R_{N}=R_{n}$ for all $n \geq N$. Thus we set $R:=R_{N} . R$ contains only finitely many elements.

Proof. Let $\Lambda_{\mathbb{C}}:=\operatorname{diag}\left(\lambda_{1}, \ldots, \lambda_{d}\right)$ and let $T$ be a regular matrix satisfying $E_{0}(\sigma):=T^{-1} \Lambda_{\mathbb{C}} T$. Let $n \geq 1$ be arbitrary. By the definition of $R_{n}$ an element $\left(\left[0, i_{1}^{*}\right],\left[r, i_{2}^{*}\right]\right) \in R_{n}$ satisfies

$$
r= \pm E_{0}(\sigma)^{n} x+\sum_{k=0}^{n-1} E_{0}(\sigma)^{k}\left(f\left(s_{k}\right)-f\left(t_{k}\right)\right),
$$


where $\left(\left[0, j_{1}^{*}\right],\left[x, j_{2}^{*}\right]\right) \in R_{0}$ and $s_{k}$ and $t_{k}$ are suffixes of strings of the shape $\sigma(j)$ for certain $j \in \mathcal{A}$. Setting $r_{k}=\left(r_{k}^{(1)}, \ldots, r_{k}^{(d)}\right):=T\left(f\left(s_{k}\right)-f\left(t_{k}\right)\right)$ $(0 \leq k \leq n-1)$ and $q=\left(q^{(1)}, \ldots, q^{(d)}\right):=T x$ this yields

$$
\begin{aligned}
\operatorname{Tr} & = \pm \Lambda_{\mathbb{C}}^{n} q+\sum_{k=0}^{n-1} \Lambda_{\mathbb{C}}^{k} r_{k} \\
& =\left( \pm \lambda_{1}^{n} q^{(1)}+\sum_{k=0}^{n-1} \lambda_{1}^{k} r_{k}^{(1)}, \ldots, \pm \lambda_{d}^{n} q^{(d)}+\sum_{k=0}^{n-1} \lambda_{d}^{k} r_{k}^{(d)}\right) .
\end{aligned}
$$

The first coordinate of $T r$ is bounded by a constant $L_{1}$ depending only on the choice of $T$ because $\left[r, j^{*}\right]$ is a unit tip. The other coordinates are bounded by a constant $L_{2}$ because $r_{k}$ and $q$ can attain only finitely many values and $\left|\lambda_{i}\right|<1$ for $2 \leq i \leq d$.

In other words, the elements $\left(\left[0, i^{*}\right],\left[r, j^{*}\right]\right) \in R_{n}$ satisfy

$$
\|\operatorname{Tr}\|_{\infty} \leq L
$$

for some absolute constant $L$. This implies that the number of elements in $R_{n}$ is uniformly bounded in $n$. Since $R_{n} \subseteq R_{n+1}$ it follows that the sequence $\left\{R_{n}\right\}$ is ultimately constant.

Corollary 3.5. Let $R_{0} \subseteq M \subseteq R$. Then $\Psi(M)=M$ implies that $M=R$.

Proof. Note that $M_{1} \subseteq M_{2}$ implies that $\Psi\left(M_{1}\right) \subseteq \Psi\left(M_{2}\right)$. Select $N$ such that $R_{N}=\Psi^{N}\left(R_{0}\right)=R$. Suppose that $\Psi(M)=\bar{M}$. Then

$$
R=\Psi^{N}\left(R_{0}\right) \subseteq \Psi^{N}(M)=M \subseteq R
$$

and we are done.

3.3. The contact graph. Before we give the exact definition of the contact graph we recall the definition of the adjacency matrix $A_{G}=\left(a_{i j}\right)$ of a finite directed graph $G$ (cf. [27, Definition 2.2.3]). Let $V=\{1, \ldots, q\}$ be the set of states of $G$. Then $A_{G}$ is a $q \times q$ matrix with $a_{i j}$ equal to the number of edges in $G$ leading form $i$ to $j$. The order of the states will play no role in our context. In particular, changing the order of the states of $G$ does not change the eigenvalues of $A_{G}$.

Definition 3.6. Let $\sigma$ be a unimodular Pisot substitution and let $\mathcal{Q}$ be defined as in (3.7). If $M \subset \mathcal{Q}$ then the contact graph $G(M)$ of $M$ is given in the following way. The states of $G(M)$ are the elements of $M$. Each of the states is an initial state. Furthermore, there exists an edge

$$
\begin{gathered}
\left(\left[0, i_{1}^{*}\right],\left[x, i_{2}^{*}\right]\right) \stackrel{P \mid Q}{\longrightarrow}\left(\left[0, j_{1}^{*}\right],\left[y, j_{2}^{*}\right]\right) \quad \text { (states written in canonical order) } \\
\text { with } P:=\left(p_{1}, i_{1}, s_{1}\right) \text { and } Q:=\left(p_{2}, i_{2}, s_{2}\right) \text { in } G(M) \text { if } \\
\left(\left[0, i_{1}^{*}\right],\left[x, i_{2}^{*}\right]\right) \in \Psi\left\{\left(\left[0, j_{1}^{*}\right],\left[y, j_{2}^{*}\right]\right)\right\}
\end{gathered}
$$


and

$$
\begin{aligned}
\sigma\left(j_{k}\right)=\left(p_{k}, i_{k}, s_{k}\right) \in \mathcal{P} \quad(1 \leq k \leq 2) \quad \text { or } \\
\sigma\left(j_{3-k}\right)=\left(p_{k}, i_{k}, s_{k}\right) \in \mathcal{P} \quad(1 \leq k \leq 2) .
\end{aligned}
$$

If the first alternative in (3.15) holds, the corresponding edge is said to be of type 1, if the second alternative holds, it is of type 2. If both alternatives hold, we also say that the edge is of type 1.

The adjacency matrix of $G(M)$ is called the contact matrix of $M$.

If $M=R$ then $\mathcal{C}:=G(R)$ is called the contact graph of $\sigma$. Its adjacency matrix is called the contact matrix of $\sigma$.

Obviously, all the graphs $G(M)$ are subgraphs of the graph $G(\mathcal{Q})$.

Remark 3.7. Note that the two alternatives we have for the edges correspond to the two alternatives in the definition of $\varphi$. In fact, inserting the definition of $\Psi$ we see that the edge (3.14) exists if either

$$
x=E_{0}(\sigma) y+f\left(s_{1}\right)-f\left(s_{2}\right) \quad \text { such that } \sigma\left(j_{k}\right)=\left(p_{k}, i_{k}, s_{k}\right) \in \mathcal{P}
$$

$(1 \leq k \leq 2)$ or

$$
x=-E_{0}(\sigma) y-f\left(s_{2}\right)+f\left(s_{1}\right) \quad \text { such that } \sigma\left(j_{3-k}\right)=\left(p_{k}, i_{k}, s_{k}\right) \in \mathcal{P}
$$

$(1 \leq k \leq 2)$. Indeed, $\left(\left[0, i_{1}^{*}\right],\left[x, i_{2}^{*}\right]\right) \in \Psi\left\{\left(\left[0, j_{1}^{*}\right],\left[y, j_{2}^{*}\right]\right)\right\}$ implies that $\left(\left[0, i_{1}^{*}\right],\left[x, i_{2}^{*}\right]\right)=$ $\varphi\left(\left[-f\left(\tilde{s}_{1}\right), \tilde{i}_{1}^{*}\right],\left[E_{0}(\sigma) y-f\left(\tilde{s}_{2}\right), \tilde{i}_{2}^{*}\right]\right)$ with $\sigma\left(j_{k}\right)=\tilde{p}_{k} \tilde{i}_{k} \tilde{s}_{k}(1 \leq k \leq 2)$. According to the definition of $\varphi$ this implies that

$$
\left(\left[0, i_{1}^{*}\right],\left[x, i_{2}^{*}\right]\right)= \begin{cases}{\left[0, \tilde{i}_{1}^{*}\right],\left[E_{0}(\sigma) y-f\left(\tilde{s}_{2}\right)+f\left(\tilde{s}_{1}\right), \tilde{i}_{2}^{*}\right]} & \text { or } \\ {\left[0, \tilde{i}_{2}^{*}\right],\left[-E_{0}(\sigma) y+f\left(\tilde{s}_{2}\right)-f\left(\tilde{s}_{1}\right), \tilde{i}_{1}^{*}\right] .} & \end{cases}
$$

The desired formulas are obtained by setting $\left(p_{k}, i_{k}, s_{k}\right)=\left(\tilde{p}_{k}, \tilde{i}_{k}, \tilde{s}_{k}\right)$ in the first case and $\left(p_{k}, i_{k}, s_{k}\right)=\left(\tilde{p}_{3-k}, \tilde{i}_{3-k}, \tilde{s}_{3-k}\right)$ in the second case $(1 \leq k \leq 2)$.

Both alternatives (type 1 and type 2) can hold for an edge simultaneously only if $\sigma\left(j_{1}\right)=\sigma\left(j_{2}\right)$ and, hence, $\left(p_{1}, i_{1}, s_{1}\right)=\left(p_{2}, i_{2}, s_{2}\right)$. This implies $j_{1}=j_{2}$. Thus we must have $x=E_{0}(\sigma) y$ and $x=-E_{0}(\sigma) y$. Since $E_{0}(\sigma)$ is a regular matrix we must therefore have $x=y=0$. Summing up we conclude that both alternatives can hold simultaneously only for an edge of the shape

$$
\left(\left[0, i^{*}\right],\left[0, i^{*}\right]\right) \stackrel{P \mid P}{\longrightarrow}\left(\left[0, j^{*}\right],\left[0, j^{*}\right]\right) .
$$

Throughout the paper we will always assume that the states of the occurring graphs are written in canonical order. It is easy to see that the edge

$$
\left(\left[0, i^{*}\right],\left[0, i^{*}\right]\right) \stackrel{P \mid Q}{\longrightarrow}\left(\left[0, j_{1}^{*}\right],\left[y, j_{2}^{*}\right]\right)
$$

exists if and only if

$$
\left(\left[0, i^{*}\right],\left[0, i^{*}\right]\right) \stackrel{Q \mid P}{\longrightarrow}\left(\left[0, j_{1}^{*}\right],\left[y, j_{2}^{*}\right]\right)
$$


exists. Thus we will always identify these two edges.

Definition 3.6 generalizes the graph $G(R)$, which was defined by Scheicher and Thuswaldner [36, 37] for lattice tilings. The contact matrix for lattice tilings first appears in Gröchenig and Haas [22].

For the Fibonacci substitution it is fairly easy to calculate the contact graph. In fact, we have $R_{0}=R_{1}=R$. Thus $R$ has 5 elements. From Figure 5 we easily see that

$$
R=\left\{\left(r_{1}, r_{1}\right),\left(r_{1}, r_{2}\right),\left(r_{1}, r_{3}\right),\left(r_{2}, r_{2}\right),\left(r_{2}, r_{4}\right)\right\}
$$

with $r_{1}=\left[0,1^{*}\right], r_{2}=\left[0,2^{*}\right], r_{3}=\left[(0,1), 1^{*}\right]$ and $r_{4}=\left[(1,-1), 1^{*}\right]$. If we draw the edges between these states according to Definition 3.6 we arrive at the graph depicted in Figure 7 ( $\varepsilon$ denotes the empty word). Observe that it contains a copy of the corresponding prefix-suffix automaton.

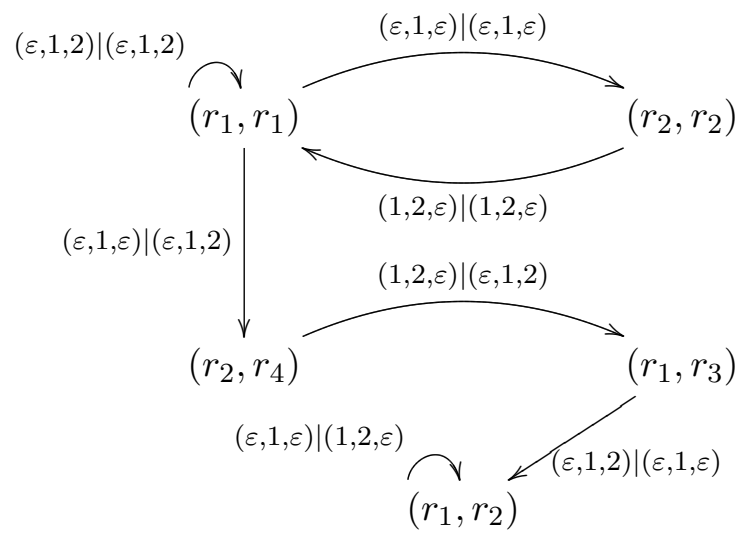

FiguRE 7 . The contact graph of the Fibonacci substitution.

The contact graph of the Tribonacci substitution will be constructed later. It is described in Theorem 6.4.

Lemma 3.8. Let $\sigma$ be a unimodular Pisot substitution and let $H$ be a subgraph of $G(\mathcal{Q})$. If from each state $\left(\left[0, i_{1}^{*}\right],\left[x, i_{2}^{*}\right]\right)$ of $H$ there leads a walk to a state $\left(\left[0, j_{1}^{*}\right],\left[y, j_{2}^{*}\right]\right) \in R_{0}$ then $H \subset \mathcal{C}=G(R)$.

Proof. By definition, $R$ contains all elements $\left(\left[0, i_{1}^{*}\right],\left[x, i_{2}^{*}\right]\right) \in \mathcal{Q}$ from which there leads a walk to a state of the shape $\left(\left[0, j_{1}^{*}\right],\left[y, j_{2}^{*}\right]\right) \in R_{0}$ in $G(\mathcal{Q})$. Since $H \subseteq G(\mathcal{Q})$ we are done.

\section{On the boundary of a tile}

Our next task is the description of the boundary of the atomic surfaces in terms of the contact graph. In what follows we need that also the limit 
of the tilings $\mathcal{I}_{n}$ defined in (3.6) for $n \rightarrow \infty$ yields a tiling. Let $X_{i}(n)$ be defined as in (3.3). Since $X_{i}(n) \rightarrow X_{i}$ this is tantamount to saying that $\mathcal{I}$ in (2.8) tiles $\mathbf{P}$. As mentioned in the introduction this is true only if the so-called super coincidence condition holds.

Definition 4.1 (cf. $[7,25])$. Let $[x, i]$ and $[y, j]$ with $x, y \in \mathbb{Z}^{d}$ and $i, j \in \mathcal{A}$ be given. We say that $[x, i]$ and $[y, j]$ have strong coincidence if there exists an $n \in \mathbb{N}$ such that $E_{1}(\sigma)^{n}[x, i] \cap E_{1}(\sigma)^{n}[y, j]$ have at least one line segment in common.

Let $\pi^{\prime}$ be the projection of $\mathbb{R}^{d}$ to $u$ along $\mathbf{P}$. We say that $[x, i]$ and $[y, j]$ have the same height if

$$
\operatorname{int}\left(\pi^{\prime}[x, i]\right) \cap \operatorname{int}\left(\pi^{\prime}[y, j]\right) \neq \emptyset .
$$

Furthermore, $\sigma$ satisfies the super coincidence condition if $[x, i]$ and $[y, j]$ have strong coincidence whenever they have the same height.

If this condition holds for $\sigma$ then [25, Theorem 1.3] yields that $\mathcal{I}$ tiles P. Together with [25, Theorem 3.3] this implies that $\left(\hat{X}_{i}(n)\right.$ is defined in $(3.2))$

$$
\lim _{n \rightarrow \infty} E_{0}(\sigma)^{n} \partial \hat{X}_{i}(n)=\partial X_{i} \quad(i \in \mathcal{A})
$$

in Hausdorff metric (cf. also [20, Chapter 8] and [6]; for the lattice tile analogue of these results we refer to Vince [43, Theorem 4.2]). Setting $\hat{X}(n):=\bigcup_{i \in \mathcal{A}} \hat{X}_{i}(n)$ an analogous identity holds for $\partial X$.

4.1. The boundaries of the approximations of $X_{i}$. For fixed $n$ the set $\hat{X}_{i}(n)$ in $(3.2)$ is the union of $(d-1)$-dimensional prisms of the shape $\pi\left[x, i^{*}\right]$. Its boundary is a union of $(d-2)$-dimensional prisms of the shape

$$
\pi\left[x_{1}, i_{1}^{*}\right] \cap \pi\left[x_{2}, i_{2}^{*}\right]
$$

Let $\mathcal{Q}$ be defined as in $(3.7)$. For $\left(\left[0, i_{1}^{*}\right],\left[x, i_{2}^{*}\right]\right) \in \mathcal{Q}$ let

$$
B\left(\left(\left[0, i_{1}^{*}\right],\left[x, i_{2}^{*}\right]\right), n\right):=\hat{X}_{i_{1}}(n) \cap\left(\hat{X}_{i_{2}}(n)+E_{0}(\sigma)^{-n} \pi(x)\right) .
$$

If $\left[0, i_{1}^{*}\right] \neq\left[x, i_{2}^{*}\right]$ then $B\left(\left(\left[0, i_{1}^{*}\right],\left[x, i_{2}^{*}\right]\right), n\right)$ consists of a union of prisms of the shape (4.2). The number of these prisms will be denoted by

$$
\Pi\left(B\left(\left(\left[0, i_{1}^{*}\right],\left[x, i_{2}^{*}\right]\right), n\right)\right) .
$$

Lemma 4.2. Let $\sigma$ be a unimodular Pisot substitution. If $\left[0, i_{1}^{*}\right] \neq\left[x, i_{2}^{*}\right]$ then for $n \geq 0$ we have

$$
\Pi\left(B\left(\left(\left[0, i_{1}^{*}\right],\left[x, i_{2}^{*}\right]\right), n\right)\right)>0 \quad \Longrightarrow \quad\left(\left[0, i_{1}^{*}\right],\left[x, i_{2}^{*}\right]\right) \in R_{n} .
$$

If $n$ is large enough such that $R_{n}=R$ then this becomes

$$
\Pi\left(B\left(\left(\left[0, i_{1}^{*}\right],\left[x, i_{2}^{*}\right], n\right)\right)>0 \quad \Longrightarrow \quad\left(\left[0, i_{1}^{*}\right],\left[x, i_{2}^{*}\right]\right) \in R .\right.
$$


This implies that

$$
\partial X_{i}(n)=\bigcup_{\substack{\left(\left[0, i^{*}\right],\left[y, j^{*}\right]\right) \in R_{n} \\\left[0, i^{*}\right] \neq\left[y, j^{*}\right]}}\left(X_{i}(n) \cap\left(X_{j}(n)+\pi(y)\right)\right) \quad(i \in \mathcal{A}, n \in \mathbb{N})
$$

Proof. We use induction on $n$ in order to prove the lemma. For $n=0$ the result is true by (3.8). Now suppose that it is true for $n-1$. Then we get

$$
\begin{aligned}
& B\left(\left(\left[0, i_{1}^{*}\right],\left[x, i_{2}^{*}\right]\right), n\right) \\
& =\hat{X}_{i_{1}}(n) \cap\left(\hat{X}_{i_{2}}(n)+E_{0}(\sigma)^{-n} \pi(x)\right) \\
& =\pi\left(\bigcup_{\left[y_{1}, j_{1}\right] \in E_{1}^{*}(\sigma)\left[0, i_{1}^{*}\right]} \bigcup_{\left[y, j^{*}\right] \in E_{1}^{*}(\sigma)^{n-1}\left[y_{1}, j_{1}^{*}\right]}\left[y, j^{*}\right]\right) \cap \\
& \pi\left(\bigcup_{\left[y_{2}, j_{2}\right] \in E_{1}^{*}(\sigma)\left[0, i_{2}^{*}\right]} \bigcup_{\left[y, j^{*}\right] \in E_{1}^{*}(\sigma)^{n-1}\left[y_{2}, j_{2}^{*}\right]}\left[y, j^{*}\right]+E_{0}(\sigma)^{-n} x\right) .
\end{aligned}
$$

Inserting the definition of $E_{1}^{*}(\sigma)$ in the first union yields

$$
\begin{aligned}
& B\left(\left(\left[0, i_{1}^{*}\right],\left[x, i_{2}^{*}\right]\right), n\right) \\
& =\pi\left(\bigcup_{i_{1} \stackrel{\left(p_{1}, i_{1}, s_{1}\right)}{\longrightarrow} j_{1}} \bigcup_{\left[y, j^{*}\right] \in E_{1}^{*}(\sigma)^{n-1}\left[E_{0}(\sigma)^{-1} f\left(s_{1}\right), j_{1}^{*}\right]}\left[y, j^{*}\right]\right) \cap \\
& \pi\left(\bigcup_{i_{2} \stackrel{\left(p_{2}, i_{2}, s_{2}\right)}{\longrightarrow} j_{2}} \bigcup_{\left[y, j^{*}\right] \in E_{1}^{*}(\sigma)^{n-1}\left[E_{0}(\sigma)^{-1} f\left(s_{2}\right), j_{2}^{*}\right]}\left[y, j^{*}\right]+E_{0}(\sigma)^{-n} x\right) \\
& =\pi\left(\bigcup_{i_{1} \stackrel{\left(p_{1}, i_{1}, s_{1}\right)}{\longrightarrow} j_{1}} \bigcup_{\left[y, j^{*}\right] \in E_{1}^{*}(\sigma)^{n-1}\left[0, j_{1}^{*}\right]}\left[y, j^{*}\right]+E_{0}(\sigma)^{-n} f\left(s_{1}\right)\right) \cap \\
& \pi\left(\bigcup_{i_{2} \stackrel{\left(p_{2}, i_{2}, s_{2}\right)}{\longrightarrow} j_{2}} \bigcup_{\left[y, j^{*}\right] \in E_{1}^{*}(\sigma)^{n-1}\left[0, j_{2}^{*}\right]}\left[y, j^{*}\right]+E_{0}(\sigma)^{-n} f\left(s_{2}\right)+E_{0}(\sigma)^{-n} x\right) .
\end{aligned}
$$


Changing the order of the unions and inserting the definition of $\hat{X}_{\ell}(n-1)$ for $\ell=j_{1}$ and $j_{2}$ yields that this is equal to

$$
\begin{aligned}
& \bigcup_{i_{1} \stackrel{\left(p_{1}, i_{1}, s_{1}\right)}{\longrightarrow} j_{1}} \bigcup_{i_{2} \stackrel{\left(p_{2}, i_{2}, s_{2}\right)}{\longrightarrow} j_{2}}\left(\left(\hat{X}_{j_{1}}(n-1)+\pi E_{0}(\sigma)^{-n} f\left(s_{1}\right)\right)\right. \\
& \left.\cap\left(\hat{X}_{j_{2}}(n-1)+\pi E_{0}(\sigma)^{-n}\left(f\left(s_{2}\right)+x\right)\right)\right) \\
& =\underset{\substack{i_{1} \stackrel{\left(p_{1}, i_{1}, s_{1}\right)}{\longrightarrow} j_{1} \\
\left[E_{0}(\sigma)^{-1}\left(f\left(s_{2}\right)-f\left(s_{1}\right)+x\right), j_{2}^{*}\right] \in S}}{i_{2} \stackrel{\left(p_{2}, i_{2}, s_{2}\right)}{\longrightarrow} j_{2}} \\
& \left.B\left(\left(\left[0, j_{1}^{*}\right],\left[E_{0}(\sigma)^{-1}\left(f\left(s_{2}\right)-f\left(s_{1}\right)+x\right), j_{2}^{*}\right]\right), n-1\right)+\pi E_{0}(\sigma)^{-n} f\left(s_{1}\right)\right)
\end{aligned}
$$

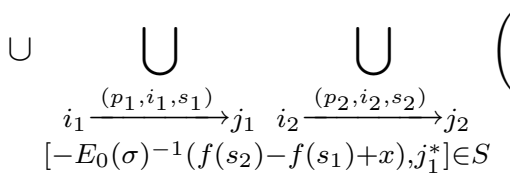

$$
\begin{aligned}
& B\left(\left(\left[0, j_{2}^{*}\right],\left[-E_{0}(\sigma)^{-1}\left(f\left(s_{2}\right)-f\left(s_{1}\right)+x\right), j_{1}^{*}\right]\right), n-1\right) \\
& \left.+\pi E_{0}(\sigma)^{-n}\left(f\left(s_{2}\right)+x\right)\right)
\end{aligned}
$$

The fact that we have to split up the double unions in two parts comes from the definition of $\varphi$. Note that one of the quantities $\left[E_{0}(\sigma)^{-1}\left(f\left(s_{2}\right)-\right.\right.$ $\left.\left.f\left(s_{1}\right)+x\right), j_{2}^{*}\right]$ and $\left[-E_{0}(\sigma)^{-1}\left(f\left(s_{2}\right)-f\left(s_{1}\right)+x\right), j_{1}^{*}\right]$ is always contained in $S$. Indeed, $E_{1}^{*}(\sigma)$ transforms a unit tip in a union of unit tips (cf. [6, Proposition 1]) and $\varphi$ transforms a pair of unit tips into a pair of unit tips 
by definition. Summing up we proved that

$$
\begin{aligned}
& \Pi\left(B\left(\left(\left[0, i_{1}^{*}\right],\left[x, i_{2}^{*}\right]\right), n\right)\right) \\
& =\Pi\left(\underset{\substack{i_{1} \underset{\left(p_{1}, i_{1}, s_{1}\right)}{\longrightarrow} j_{1} \underset{i_{2}}{\left[E_{0}(\sigma)^{-1}\left(f\left(s_{2}\right)-f\left(s_{1}\right)+x\right), j_{2}^{*}\right] \in S} j_{2} \\
\bigcup}}{\bigcup}\right. \\
& \left.B\left(\left(\left[0, j_{1}^{*}\right],\left[E_{0}(\sigma)^{-1}\left(f\left(s_{2}\right)-f\left(s_{1}\right)+x\right), j_{2}^{*}\right]\right), n-1\right)+\pi E_{0}(\sigma)^{-n} f\left(s_{1}\right)\right) \\
& \cup \bigcup_{\substack{i_{1} \stackrel{\left(p_{1}, i_{1}, s_{1}\right)}{\longrightarrow} j_{1} \\
\left[-E_{0}(\sigma)^{-1}\left(f\left(s_{2}\right)-f\left(s_{1}\right)+x\right), j_{1}^{*}\right] \in S}} \bigcup_{i_{2} \stackrel{\left(p_{2}, i_{2}, s_{2}\right)}{\longrightarrow} j_{2}}( \\
& B\left(\left(\left[0, j_{2}^{*}\right],\left[-E_{0}(\sigma)^{-1}\left(f\left(s_{2}\right)-f\left(s_{1}\right)+x\right), j_{1}^{*}\right]\right), n-1\right)+ \\
& \left.\left.\pi E_{0}(\sigma)^{-n}\left(f\left(s_{2}\right)+x\right)\right)\right) .
\end{aligned}
$$

By the induction hypothesis this is greater than zero if and only if at least one of the elements

$$
\left(\left[0, j_{1}^{*}\right],\left[E_{0}(\sigma)^{-1}\left(f\left(s_{2}\right)-f\left(s_{1}\right)+x\right), j_{2}^{*}\right]\right)
$$

or

$$
\left(\left[0, j_{2}^{*}\right],\left[-E_{0}(\sigma)^{-1}\left(f\left(s_{2}\right)-f\left(s_{1}\right)+x\right), j_{1}^{*}\right]\right)
$$

is contained in $R_{n-1}$. If this is the case then by the definition of $R_{n}$ in terms of $R_{n-1}$ we have $\left(\left[0, i_{1}^{*}\right],\left[x, i_{2}^{*}\right]\right) \in R_{n}$ and we are done.

4.2. A set equation for the boundaries of $X_{i}$. In the remaining part of the paper we will always assume that $\sigma$ satisfies the super coincidence condition. We are now in a position to give a description of the boundaries of $X_{i}(i \in \mathcal{A})$ as a graph directed system.

Let $\epsilon:\left(\left[0, i_{1}^{*}\right],\left[x, i_{2}^{*}\right]\right) \stackrel{\left(p_{1}, i_{1}, s_{1}\right) \mid\left(p_{2}, i_{2}, s_{2}\right)}{\longrightarrow}\left(\left[0, j_{1}^{*}\right],\left[y, j_{2}^{*}\right]\right)$ be an edge in $\mathcal{C}$ (the states are written in canonical order) and set

$$
F(\epsilon):= \begin{cases}f\left(s_{1}\right) & \text { if } \epsilon \text { is of type 1, } \\ f\left(s_{2}\right)+x & \text { if } \epsilon \text { is of type 2. }\end{cases}
$$

Note that for those edges, for which both alternatives of Definition 3.6 hold both alternatives in the definition of $F(\varepsilon)$ coincide. Indeed, in view of 
Remark 3.7 we have $x=0$ and $s_{1}=s_{2}$ in this case. An easy calculation shows that $F(\epsilon)$ is the same for the identified edges (3.16) and (3.17).

We define the non-empty compact sets $C(v) \subset \mathbf{P}(v \in R)$ by the following graph directed system:

$$
C\left(v_{1}\right)=\bigcup_{\epsilon: v_{1} \rightarrow v_{2}} E_{0}(\sigma) C\left(v_{2}\right)+\pi F(\epsilon) .
$$

The union is extended over all outgoing edges of $v_{1}$ in the contact graph $\mathcal{C}$. In particular, $C(v)$ is empty if the state $v$ has no outgoing edges.

Theorem 4.3. Let $\sigma$ be a unimodular Pisot substitution which satisfies the super coincidence condition and let $X$ and $\left\{X_{i}\right\}_{i \in \mathcal{A}}$ be the associated atomic surfaces. The boundaries of $X$ and $X_{i}(i \in \mathcal{A})$ can be characterized as follows.

$$
\begin{aligned}
& \partial X= \bigcup_{\substack{\left(\left[0, i^{*}\right],\left[y, j^{*}\right]\right) \in R \\
y \neq 0}} C\left(\left(\left[0, i^{*}\right],\left[y, j^{*}\right]\right)\right), \\
& \partial X_{i}=\bigcup_{\substack{\left(\left[0, i^{*}\right],\left[y, j^{*}\right]\right) \in R \\
i \text { fixed, }\left[0, i^{*}\right] \neq\left[y, j^{*}\right]}} C\left(\left(\left[0, i^{*}\right],\left[y, j^{*}\right]\right)\right) \quad(i \in \mathcal{A}),
\end{aligned}
$$

where $C(v)(v \in R)$ is uniquely defined by the graph directed system (4.4).

Remark 4.4. Note that equation (4.4) is related to the dual map $E_{2}^{*}(\sigma)$ of the two dimensional realization $E_{2}(\sigma)$ of $\sigma$ in Sano et al. [35]. With help of this dual map one also gets a parametrization of the boundary of $X_{i}$ (cf. [35, Proposition 3.1]). This technique was used in Ito-Kimura [24] to determine the Hausdorff dimension of the boundary of the classical Rauzy fractal.

Proof. Let $N$ be large enough such that $R_{N}=R$. Then by the same reasoning as in the proof of Lemma 4.2 we get

$$
B\left(v_{1}, n\right)=\bigcup_{\epsilon: v_{1} \rightarrow v_{2}} B\left(v_{2}, n-1\right)+\pi E_{0}(\sigma)^{-n} F(\epsilon) .
$$

Thus $E_{0}(\sigma)^{n} B(v, n)$ converges to $C(v)$ for $n \rightarrow \infty$ in Hausdorff metric (cf. [18, Theorem 2.6 and Chapter 3]), i.e.

$$
C(v)=\lim _{n \rightarrow \infty} E_{0}(\sigma)^{n} B(v, n)
$$

holds for each $v \in R$. Since

$$
\partial \hat{X}(n)=\bigcup_{\substack{\left(\left[0, i_{1}^{*}\right],\left[x, i_{2}^{*}\right]\right) \in \mathcal{Q} \\ x \neq 0}} B\left(\left(\left[0, i_{1}\right],\left[x, i_{2}^{*}\right]\right), n\right)
$$


Lemma 4.2 implies that

$$
\partial \hat{X}(n)=\bigcup_{\substack{\left(\left[0, i_{1}^{*}\right],\left[x, i_{2}^{*}\right]\right) \in R \\ x \neq 0}} B\left(\left(\left[0, i_{1}\right],\left[x, i_{2}^{*}\right]\right), n\right) .
$$

Note that if $A_{n} \rightarrow A, B_{n} \rightarrow B$ and $A_{n} \cup B_{n} \rightarrow C$ in Hausdorff metric then $C=A \cup B$. Thus multiplying by $E_{0}(\sigma)^{n}$ and taking limits in (4.8), (4.1) yields (4.6). (4.5) is shown analogously.

For $v \in R$ let $C(v)$ be defined as in (4.4). Using (4.7), (4.3) and (4.1) we see that

$$
\begin{aligned}
C\left(\left(\left[0, i^{*}\right],\left[0, i^{*}\right]\right)\right) & =\lim _{n \rightarrow \infty} E_{0}(\sigma)^{n} B\left(\left[0, i^{*}\right],\left[0, i^{*}\right], n\right) \\
& =\lim _{n \rightarrow \infty} E_{0}(\sigma)^{n} \hat{X}_{i}(n) \\
& =X_{i} .
\end{aligned}
$$

Thus the state $\left(\left[0, i^{*}\right],\left[0, i^{*}\right]\right)$ in $\mathcal{C}$ corresponds to the set $X_{i}$. Suppose that there exists an edge $\left(\left[0, i_{1}^{*}\right],\left[x, i_{2}^{*}\right]\right) \rightarrow\left(\left[0, j^{*}\right],\left[0, j^{*}\right]\right)$ in $\mathcal{C}$. Then, by $(4.4)$ the set $C\left(\left(\left[0, i_{1}^{*}\right],\left[x, i_{2}^{*}\right]\right)\right)$ contains a shrinked copy of $X_{j}$. Thus the set $C\left(\left(\left[0, i_{1}^{*}\right],\left[x, i_{2}^{*}\right]\right)\right)$ has inner points because $X_{j}$ has inner points by Proposition 2.3. But since by assumption there are no overlaps in the tiling $\mathcal{I}$ this implies that $i_{1}=i_{2}$ and $x=0$ and, hence, $C\left(\left(\left[0, i_{1}^{*}\right],\left[x, i_{2}^{*}\right]\right)\right)=X_{i_{1}}$. Thus we may omit the states $\left(\left[0, i^{*}\right],\left[0, i^{*}\right]\right)$ from $\mathcal{C}$ without affecting $(4.4)$ for those sets $C(v)$ which are subsets of the boundary of one of the sets $X_{i}$. From the resulting subgraph we may successively omit all states having no outgoing edge because the sets $C(v)$ corresponding to these states $v$ are empty. Thus we loose nothing by omitting these states. This motivates the following definition.

Definition 4.5. Let $\mathcal{C}$ be the contact graph. Delete the states $\left(\left[0, i^{*}\right],\left[0, i^{*}\right]\right)$ from $\mathcal{C}$ and from the resulting subgraph successively delete all states having no outgoing edges. The boundary graph emerging from this process will be denoted by $\mathcal{C}_{\partial}$.

In the sequel we will need the following definitions (cf. for instance [27, p. 37]). Let $G$ be a directed graph. We call a state $v$ of $G$ a stranding state if no edges start at $v$ or no edges terminate at $v$. $G$ is called essential if it contains no stranding states. The graph emerging from $G$ by removing all stranding states is called the essential part of $G$.

Let $\mathcal{C}_{\partial}^{\prime}$ be the essential part of $\mathcal{C}_{\partial}$ (i.e. remove all states of $\mathcal{C}_{\partial}$ having no incoming edges) and let $V \in \mathcal{C}_{\partial}$ be an arbitrary state. Since each state of $\mathcal{C}_{\partial}$ has outgoing edges we conclude that each walk starting at $V$ arrives at a state $V^{\prime} \in \mathcal{C}_{\partial}^{\prime}$ after finitely many steps. 
4.3. Using prefixes instead of suffixes. Let $\sigma$ be a unimodular Pisot substitution. All the definitions and results of this paper can be established also by working with prefixes instead of suffixes. In this case the set equation in (2.5) for the atomic surfaces $X_{i}^{\prime}$ associated to $\sigma$ reads

$$
X_{i}^{\prime}=\bigcup_{\substack{(p, i, s), j \\(p, i, s)}}^{\longrightarrow} E_{0}(\sigma) X_{j}^{\prime}-\pi f(p)
$$

where

$$
X_{i}^{\prime}=X_{i}+\pi\left(e_{i}\right) \quad(i \in \mathcal{A})
$$

(cf. [6, Lemma 8]). Moreover, the following slight modifications are needed (cf. [6]). Firstly, we have to set $[x, i]:=\left\{x+\theta e_{i}: \theta \in[0,1]\right\}$ and

$\left.\left[x, i^{*}\right]:=\left\{x+e_{i}+\theta_{1} e_{1}+\ldots+\theta_{i-1} e_{i-1}+\theta_{i+1} e_{i+1}+\ldots+\theta_{d} e_{d}\right): \theta_{i} \in[0,1]\right\}$.

The stepped surface $S$ has to be

$$
S:=\left\{\left[x, i^{*}\right]: x \in \mathbb{Z}^{d}, 1 \leq i \leq d \text { such that } x \in \mathbf{P}^{<0} \text { and } x+e_{i} \in \mathbf{P}^{\geq 0}\right\}
$$

in the prefix setting, the set $S_{0}$ is defined by $S_{0}:=\left\{\left[-e_{i}, i^{*}\right]: i \in \mathcal{A}\right\} \subset S$. The definitions of $\varphi$ and $\mathcal{Q}$ have to be changed accordingly. For the one dimensional geometric realization $E_{1}(\sigma)$ and its dual $E_{1}^{*}(\sigma)$ we have

$$
E_{1}(\sigma)[y, j]:=E_{0}(\sigma) y+\bigcup_{\substack{(p, i, s), i \\(p, i, s)}}^{\longrightarrow} j
$$

and

$$
E_{1}^{*}(\sigma)\left[x, i^{*}\right]:=E_{0}(\sigma)^{-1} x-\bigcup_{\substack{(p, i, s), j \\(p, i, s)}}^{\longrightarrow}\left[E_{0}(\sigma)^{-1} f(p), j^{*}\right] .
$$

These modifications cause that different pairs of unit tips pair $(d-2)$ dimensional faces with each other. However, the structure of the resulting contact graph is of course the same because both constructions are equivalent.

\section{Hausdorff and box counting dimension of the boundary}

Throughout this section we assume that the super coincidence condition is fulfilled for the substitutions under discussion. By Theorem 4.3 we know that $\partial X$ is a graph directed self-affine set directed by $\mathcal{C}_{\partial}$. We will now determine the box counting dimension of $\partial X$ and $\partial X_{i}(i \in \mathcal{A})$. If $\partial X$ is even graph directed self-similar, according to a general theory (cf. for instance Falconer [18, Chapter 3]) the box counting dimension coincides with the Hausdorff dimension. 
5.1. Preparation: construction of covers. We will use the notation $W_{L}\left(V, V^{\prime}\right)$ for all walks from a state $V$ to a state $V^{\prime}$ of length $L$ in $\mathcal{C}_{\partial}$ and $W_{L}(V)$ for all walks in $\mathcal{C}_{\partial}$ starting at $V$. The set of all walks of length $L$ in $\mathcal{C}_{\partial}$ will be denoted by $W_{L}$.

Let

$$
\epsilon:\left(\left[0, i_{1}^{*}\right],\left[x, i_{2}^{*}\right]\right) \stackrel{\left(p_{1}, i_{1}, s_{1}\right) \mid\left(p_{2}, i_{2}, s_{2}\right)}{\longrightarrow}\left(\left[0, j_{1}^{*}\right],\left[y, j_{2}^{*}\right]\right)
$$

(states written in canonical order) be an edge in $\mathcal{C}_{\partial}$ and define

$$
\begin{aligned}
\chi_{\epsilon}: \mathbf{P} & \rightarrow \mathbf{P}, \\
t & \mapsto E_{0}(\sigma) t+\pi F(\epsilon) .
\end{aligned}
$$

More generally, let

$$
w: V_{L} \stackrel{P_{L} \mid Q_{L}}{\longrightarrow} V_{L-1} \stackrel{P_{L-1} \mid Q_{L-1}}{\longrightarrow} \cdots \stackrel{P_{1} \mid Q_{1}}{\longrightarrow} V_{0}
$$

be a walk in $W_{L}$ and set $V_{\ell}:=\left(\left[0, i_{\ell}^{*}\right],\left[x_{\ell}, j_{\ell}^{*}\right]\right)$ (written in canonical order). Denote the edge $V_{\ell} \stackrel{P_{\ell} \mid Q_{\ell}}{\longrightarrow} V_{\ell-1}$ by $\epsilon_{\ell}$. Then set

$$
\begin{aligned}
\chi_{w}: \mathbf{P} & \rightarrow \mathbf{P}, \\
t & \mapsto \chi_{\epsilon_{L}} \circ \cdots \circ \chi_{\epsilon_{1}}(t) .
\end{aligned}
$$

Note that the functions $\chi_{w}$ are contractions since $E_{0}(\sigma)$ is a contraction on $\mathbf{P}$.

We associate two walks of the prefix-suffix automaton $\Gamma_{\sigma}$ (see Definition 2.2) to the walk $w$ in (5.2) in the following way. First we set

$$
\begin{aligned}
& \left(p_{1}, i_{1}, s_{1}\right):= \begin{cases}P_{1} & \text { if } \epsilon_{1} \text { is of type } 1, \\
Q_{1} & \text { if } \epsilon_{1} \text { is of type 2, }\end{cases} \\
& \left(q_{1}, j_{1}, t_{1}\right):= \begin{cases}P_{1} & \text { if } \epsilon_{1} \text { is of type } 2, \\
Q_{1} & \text { if } \epsilon_{1} \text { is of type } 1 .\end{cases}
\end{aligned}
$$

For $2 \leq \ell \leq L$ we define $\left(p_{\ell}, i_{\ell}, s_{\ell}\right)$ and $\left(q_{\ell}, j_{\ell}, t_{\ell}\right)$ iteratively as follows. If we have defined $\left(p_{\ell-1}, i_{\ell-1}, s_{\ell-1}\right):=P_{\ell-1}$ then

$$
\begin{aligned}
& \left(p_{\ell}, i_{\ell}, s_{\ell}\right):= \begin{cases}P_{\ell} & \text { if } \epsilon_{\ell} \text { is of type } 1, \\
Q_{\ell} & \text { if } \epsilon_{\ell} \text { is of type 2, }\end{cases} \\
& \left(q_{\ell}, j_{\ell}, t_{\ell}\right):= \begin{cases}P_{\ell} & \text { if } \epsilon_{\ell} \text { is of type } 2, \\
Q_{\ell} & \text { if } \epsilon_{\ell} \text { is of type } 1 .\end{cases}
\end{aligned}
$$


If, at the contrary, we have defined $\left(p_{\ell-1}, i_{\ell-1}, s_{\ell-1}\right):=Q_{\ell-1}$ then

$$
\begin{aligned}
& \left(p_{\ell}, i_{\ell}, s_{\ell}\right):= \begin{cases}Q_{\ell} & \text { if } \epsilon_{\ell} \text { is of type 1, } \\
P_{\ell} & \text { if } \epsilon_{\ell} \text { is of type 2, }\end{cases} \\
& \left(q_{\ell}, j_{\ell}, t_{\ell}\right):= \begin{cases}Q_{\ell} & \text { if } \epsilon_{\ell} \text { is of type 2, } \\
P_{\ell} & \text { if } \epsilon_{\ell} \text { is of type } 1 .\end{cases}
\end{aligned}
$$

The reason why we defined these quantities in this way is that according to the definition of $\mathcal{C}_{\partial}$ the sequences $\left(p_{\ell}, i_{\ell}, s_{\ell}\right)_{L \geq \ell \geq 1}$ and $\left(q_{\ell}, j_{\ell}, t_{\ell}\right)_{L \geq \ell \geq 1}$ give rise to walks in $\Gamma_{\sigma}$ (with $L \geq \ell \geq 1$ we mean that the walk starts with $\left(p_{L}, i_{L}, s_{L}\right)$ and ends with $\left.\left(p_{1}, i_{1}, s_{1}\right)\right)$.

Lemma 5.1. Let $w \in W_{L}$ be the walk in (5.2) and associate the sequences $\left(p_{\ell}, i_{\ell}, s_{\ell}\right)_{L \geq \ell \geq 1}$ and $\left(q_{\ell}, j_{\ell}, t_{\ell}\right)_{L \geq \ell \geq 1}$ to it as above. If $P_{L}=\left(p_{L}, i_{L}, s_{L}\right)$ then

$$
x_{0}=E_{0}(\sigma)^{-L} x_{L}+\sum_{\ell=1}^{L} E_{0}(\sigma)^{-\ell}\left(f\left(t_{\ell}\right)-f\left(s_{\ell}\right)\right),
$$

if $P_{L}=\left(q_{L}, j_{L}, t_{L}\right)$ then

$$
x_{0}=-E_{0}(\sigma)^{-L} x_{L}+\sum_{\ell=1}^{L} E_{0}(\sigma)^{-\ell}\left(f\left(t_{\ell}\right)-f\left(s_{\ell}\right)\right) .
$$

Proof. This can easily be proved by induction on $L$. If (5.3) holds for a certain $L \in \mathbb{N}$ we will say for short that $A_{1}(L)$ holds. Similar, if (5.4) is true for a certain $L \in \mathbb{N}$ we will say that $A_{2}(L)$ holds.

Let $V_{\ell}$ be defined as above. We start with $L=1$. If $\epsilon_{1}$ is of type 1 then $P_{1}=\left(p_{1}, i_{1}, s_{1}\right)$ and $Q_{1}=\left(q_{1}, j_{1}, t_{1}\right)$. Using the definition of $\mathcal{C}_{\partial}$ we get $x_{0}=$ $E_{0}(\sigma)^{-1} x_{1}+E_{0}(\sigma)^{-1}\left(f\left(t_{1}\right)-f\left(s_{1}\right)\right)$. If $\epsilon_{1}$ is of type 2 we have $P_{1}=\left(q_{1}, j_{1}, t_{1}\right)$ and $Q_{1}=\left(p_{1}, i_{1}, s_{1}\right)$. This yields $x_{0}=-E_{0}(\sigma)^{-1} x_{1}+E_{0}(\sigma)^{-1}\left(f\left(t_{1}\right)-f\left(s_{1}\right)\right)$.

Now we turn to the induction step. We will prove $A_{1}(L)$ and $A_{2}(L)$ assuming $A_{1}(L-1)$ and $A_{2}(L-1)$ : Suppose first that $P_{L-1}=\left(p_{L-1,} i_{L-1}, s_{L-1}\right)$ and that $A_{1}(L-1)$ is true. If $\epsilon_{L}$ is of type 1 then $P_{L}=\left(p_{L}, i_{L}, s_{L}\right)$ and $Q_{L}=\left(q_{L}, j_{L}, t_{L}\right)$. Applying the definition of $\mathcal{C}_{\partial}$ this implies that

$$
x_{L-1}=E_{0}(\sigma)^{-1} x_{L}+E_{0}(\sigma)^{-1}\left(f\left(t_{L}\right)-f\left(s_{L}\right)\right) .
$$

Inserting this in $A_{1}(L-1)$ proves $A_{1}(L)$ in this case. If $\epsilon_{L}$ is of type 2 then $P_{L}=\left(q_{L}, j_{L}, t_{L}\right)$ and $Q_{L}=\left(p_{L}, i_{L}, s_{L}\right)$. The proof of $A_{2}(L)$ is done in the same way in this case.

Now assume that $P_{L-1}=\left(q_{L-1,} j_{L-1}, t_{L-1}\right)$ holds and that $A_{2}(L-1)$ is true. If $\epsilon_{L}$ is of type 1 then $P_{L}=\left(q_{L}, j_{L}, t_{L}\right)$ and $Q_{L}=\left(p_{L}, i_{L}, s_{L}\right)$. Applying the definition of $\mathcal{C}_{\partial}$ this implies that

$$
x_{L-1}=E_{0}(\sigma)^{-1} x_{L}+E_{0}(\sigma)^{-1}\left(f\left(s_{L}\right)-f\left(t_{L}\right)\right) .
$$


Inserting this in $A_{2}(L-1)$ proves $A_{2}(L)$ in this case. If $\epsilon_{L}$ is of type 2 then $P_{L}=\left(p_{L}, i_{L}, s_{L}\right)$ and $Q_{L}=\left(q_{L}, j_{L}, t_{L}\right)$. The proof of $A_{1}(L)$ is done in the same way in this case.

Let $\rho>0$ and let $\mathcal{B}$ be an arbitrary ball of radius $\rho$. Iterating $(2.5) L$ times, multiplying by $E_{0}(\sigma)^{-L}$ and observing that the sets $X_{j}$ are compact and have non-empty interior by Proposition 2.3, we see that there is a constant $c_{\rho}$ independent of $L$ which bounds the number of walks $\left(q_{\ell}, j_{\ell}, t_{\ell}\right)_{L \geq \ell \geq 1}$ of $\Gamma_{\sigma}$ having length $L$ and satisfying

$$
\sum_{\ell=1}^{L} E_{0}(\sigma)^{-\ell} f\left(t_{\ell}\right) \in \mathcal{B}
$$

Lemma 5.2. The number of walks in $W_{L}$ corresponding to a given walk $\left(p_{\ell}, i_{\ell}, s_{\ell}\right)_{L \geq \ell \geq 1}$ of $\Gamma_{\sigma}$ is uniformly bounded in $L$.

Proof. Let $w$ be defined as in (5.2). By Lemma 5.1 we have

$$
x_{0}= \pm E_{0}(\sigma)^{-L} x_{L}+\sum_{\ell=1}^{L} E_{0}(\sigma)^{-\ell}\left(f\left(t_{\ell}\right)-f\left(s_{\ell}\right)\right) .
$$

Here $s_{\ell}$ is fixed and for $x_{0}$ and $x_{L}$ we have at most $\left|\mathcal{C}_{\partial}\right|$ choices. The result follows from the remark preceding this lemma.

Lemma 5.3. Let $w \in W_{L}$ be as in (5.2) and associate $\left(p_{\ell}, i_{\ell}, s_{\ell}\right)_{L \geq \ell \geq 1}$ and $\left(q_{\ell}, j_{\ell}, t_{\ell}\right)_{L \geq \ell \geq 1}$ to it as above. Then

$$
\chi_{w}(0)= \begin{cases}\pi \sum_{\ell=1}^{L} E_{0}(\sigma)^{L-\ell} f\left(s_{\ell}\right) & \text { if } P_{L}=\left(p_{L}, i_{L}, s_{L}\right), \\ \pi\left(x_{L}+\sum_{\ell=1}^{L} E_{0}(\sigma)^{L-\ell} f\left(s_{\ell}\right)\right) & \text { if } P_{L}=\left(q_{L}, j_{L}, t_{L}\right) .\end{cases}
$$

Proof. The proof is done by induction. Let $L=1$. If $\epsilon_{1}$ is of type 1 then $\chi_{\epsilon_{1}}(0)=\pi f\left(s_{1}\right)$, if it is of type 2 then $\chi_{\epsilon_{1}}(0)=\pi\left(f\left(s_{1}\right)+x_{1}\right)$.

We proceed with the induction step. Let $w^{\prime}$ be the walk emerging from $w$ by deleting the state $V_{L}$. If we assume that $P_{L-1}=\left(p_{L-1}, i_{L-1}, s_{L-1}\right)$ then by the induction hypothesis

$$
\chi_{w^{\prime}}(0)=\pi \sum_{\ell=1}^{L-1} E_{0}(\sigma)^{L-1-\ell} f\left(s_{\ell}\right) .
$$

If $\epsilon_{L}$ is of type 1 , i.e. $P_{L}=\left(p_{L}, i_{L}, s_{L}\right)$, then, since $F\left(\epsilon_{L}\right)=f\left(s_{L}\right)$,

$$
\chi_{w}(0)=\chi_{\epsilon_{L}} \circ \chi_{w^{\prime}}(0)=\pi \sum_{\ell=1}^{L} E_{0}(\sigma)^{L-\ell} f\left(s_{\ell}\right) .
$$


If $\epsilon_{L}$ is of type 2, i.e. $P_{L}=\left(q_{L}, j_{L}, t_{L}\right)$, then $F\left(\epsilon_{L}\right)=f\left(s_{L}\right)+x_{L}$ and the result follows analogously.

If we assume that $P_{L-1}=\left(q_{L-1}, j_{L-1}, t_{L-1}\right)$ then by the induction hypothesis and by Lemma 5.1

$$
\begin{aligned}
\chi_{w^{\prime}}(0) & =\pi\left(x_{L-1}+\sum_{\ell=1}^{L-1} E_{0}(\sigma)^{L-1-\ell} f\left(s_{\ell}\right)\right) \\
& =\pi\left(-E_{0}(\sigma)^{L-1} x_{0}+\sum_{\ell=1}^{L-1} E_{0}(\sigma)^{L-1-\ell} f\left(t_{\ell}\right)\right) .
\end{aligned}
$$

If $\epsilon_{L}$ is of type 1, i.e. $P_{L}=\left(q_{L}, j_{L}, t_{L}\right)$, then, since $F\left(\epsilon_{L}\right)=f\left(t_{L}\right)$,

$$
\chi_{w}(0)=\chi_{\epsilon_{L}} \circ \chi_{w^{\prime}}(0)=\pi\left(-E_{0}(\sigma)^{L} x_{0}+\sum_{\ell=1}^{L} E_{0}(\sigma)^{L-\ell} f\left(t_{\ell}\right)\right) .
$$

and the result follows from Lemma 5.1. If $\epsilon_{L}$ is of type 2, i.e. $P_{L}=$ $\left(p_{L}, i_{L}, s_{L}\right)$, the result follows analogously.

Lemma 5.4. Let $Z$ with $\operatorname{int}(Z) \supset X$ be a closed $(d-1)$-dimensional cube and $L \in \mathbb{N}$. Then there is a constant $\nu$ independent of $L$ such that the collection $\left\{\chi_{w}(Z): w \in W_{L}\right\}$ covers each point in $\mathbb{R}^{d-1}$ at most $\nu$ times.

Proof. Let $L$ be arbitrary, but fixed. We will prove the assertion for $E_{0}(\sigma)^{-L} \chi_{w}(Z)$ instead of $\chi_{w}(Z)$. Because $E_{0}(\sigma)$ is regular, this does not make a difference. In what follows let $\left(p_{\ell}, i_{\ell}, s_{\ell}\right)_{L \geq \ell \geq 1}$ and $\left(q_{\ell}, j_{\ell}, t_{\ell}\right)_{L \geq \ell \geq 1}$ be walks in $\Gamma_{\sigma}$. Since $Z$ is bounded, the remark preceding Lemma 5.2 assures that there exists an absolute constant $c_{1}$ such that $Z+\pi \sum_{\ell=1}^{L} E_{0}(\sigma)^{-\ell} f\left(t_{\ell}\right)$ has non-empty intersection with at most $c_{1}$ sets of the shape

$$
X_{j}+\pi \sum_{\ell=1}^{L} E_{0}(\sigma)^{-\ell} f\left(s_{\ell}\right) .
$$

From the same fact it follows that there even exists an absolute constant $c_{2}$ such that $Z+\pi \sum_{\ell=1}^{L} E_{0}(\sigma)^{-\ell} f\left(t_{\ell}\right)$ has non-empty intersection with at most $c_{2}$ sets of the shape $Z+\pi \sum_{\ell=1}^{L} E_{0}(\sigma)^{-\ell} f\left(s_{\ell}\right)$. Note that

$$
E_{0}(\sigma)^{-L} \chi_{w}(Z)= \begin{cases}Z+\pi \sum_{\ell=1}^{L} E_{0}(\sigma)^{L-\ell} f\left(s_{\ell}\right) & \text { or } \\ Z+\pi\left(x_{L}+\sum_{\ell=1}^{L} E_{0}(\sigma)^{L-\ell} f\left(s_{\ell}\right)\right) . & \end{cases}
$$

By Lemma 5.2 there is an absolute constant $c_{3}$ which bounds the number of $w \in W_{L}$ corresponding to a given walk $\left(p_{\ell}, i_{\ell}, s_{\ell}\right)_{L \geq \ell \geq 1}$ of $\Gamma_{\sigma}$. Furthermore, there are only $\left|\mathcal{C}_{\partial}\right|$ choices for $x_{L}$. This implies the result. 
Let $\Lambda$ and $T_{\mathbf{P}}$ be defined as in Subsection 2.3. The dimension calculations are done for the sets $C(v)$ with $v \in \mathcal{C}_{\partial}$ defined in (4.4). Since it is more convenient to work with affine transformations whose linear part is in diagonal form we work with the sets

$$
D(v):=T_{\mathbf{P}} C(v)
$$

From (4.4) we immediately get that

$$
D\left(v_{1}\right)=\bigcup_{\epsilon: v_{1} \rightarrow v_{2}} \Lambda D\left(v_{2}\right)+T_{\mathbf{P}} \pi F(\epsilon)
$$

Let $\epsilon$ be an edge in $\mathcal{C}_{\partial}$ as in (5.1). Then we set

$$
\psi_{\epsilon}(t):=\Lambda t+T_{\mathbf{P}} \pi F(\epsilon)
$$

More generally, for a walk $w$ in $\mathcal{C}_{\partial}$ consisting of the edges $\epsilon_{L}, \ldots, \epsilon_{1}$ we write

$$
\psi_{w}=\psi_{\epsilon_{L}} \circ \cdots \circ \psi_{\epsilon_{1}} .
$$

The following is an immediate consequence of Lemma 5.4.

Lemma 5.5. Let $Y=T_{\mathbf{P}} Z$ with $Z$ as in Lemma 5.4, i.e. $\operatorname{int}(Y) \supset T_{\mathbf{P}} X$, and $L \in \mathbb{N}$. Then there is a constant $\nu$ independent of $L$ such that the collection $\left\{\psi_{w}(Y): w \in W_{L}\right\}$ covers each point in $\mathbb{R}^{d-1}$ at most $\nu$ times.

5.2. Dimension calculations. Let $g: \mathbb{R}^{d-1} \rightarrow \mathbb{R}^{d-1}$ be a contractive linear map with singular values $\alpha_{1}(g) \geq \cdots \geq \alpha_{d-1}(g)>0$. The singular value function $\phi^{\kappa}(g)$ is defined for each $0 \leq \kappa \leq d-1$ by

$$
\phi^{\kappa}(g)=\alpha_{1}(g) \alpha_{2}(g) \cdots \alpha_{m-1}(g) \alpha_{m}(g)^{\kappa-m+1}
$$

where $m \in \mathbb{N}$ is given by $m-1<\kappa \leq m$.

If the essential part $\mathcal{C}_{\partial}^{\prime}$ of $\mathcal{C}_{\partial}$ is strongly connected then for each $V \in \mathcal{C}_{\partial}$ there exists a constant $C>0$ such that

$$
\left|W_{L}(V)\right| \sim C|\mu|^{L}
$$

where $\mu$ is an eigenvalue of the adjacency matrix of $\mathcal{C}_{\partial}$ having largest modulus. For $V \in \mathcal{C}_{\partial}^{\prime}$ formula (5.5) follows from the Perron-Frobenius Theorem (cf. [27, Theorem 4.2.3]). For $V \in \mathcal{C}_{\partial} \backslash \mathcal{C}_{\partial}^{\prime}$ formula (5.5) is still true because - as mentioned after Definition 4.5 - each walk starting at $V$ leads to a state $V^{\prime} \in \mathcal{C}_{\partial}^{\prime}$ after finitely many steps.

Let $\lambda^{\prime}$ be one of the eigenvalues of $E_{0}(\sigma)$ which is smallest in modulus (we have $\lambda^{\prime} \neq 0$ because $E_{0}(\sigma)$ is irreducible). Since all the contractions 
$\psi_{w}$ with $w \in W_{L}$ have the same linear part $\Lambda^{L}$ we get

$$
\begin{aligned}
\lim _{L \rightarrow \infty}\left(\sum_{w \in W_{L}(V)} \phi^{\kappa}\left(\psi_{w}\right)\right)^{1 / L} & =\lim _{L \rightarrow \infty}\left(\sum_{w \in W_{L}(V)} \phi^{\kappa}\left(\Lambda^{L}\right)\right)^{1 / L} \\
& =\lim _{L \rightarrow \infty}\left(\sum_{w \in W_{L}(V)}\left(\lambda^{-1}\left|\lambda^{\prime}\right|^{\kappa-d+1}\right)^{L}\right)^{1 / L} \\
& =\lambda^{-1}\left|\lambda^{\prime}\right|^{\kappa-d+1}|\mu|
\end{aligned}
$$

for $d-2 \leq \kappa \leq d-1$. Let $d_{\sigma}$ be the uniquely defined number $\kappa$ for which this quantity is equal to 1 . Then

$$
d_{\sigma}=d-1+\frac{\log \lambda-\log |\mu|}{\log \left|\lambda^{\prime}\right|}
$$

We are now in a position to prove the following results.

Proposition 5.6. Let $d \geq 2$ and let $X$ and $X_{i}(i \in \mathcal{A})$ be the atomic surfaces for a unimodular Pisot substitution $\sigma$ fulfilling the super coincidence condition. If the essential part of the boundary graph $\mathcal{C}_{\partial}$ is strongly connected we have the estimates

$$
\operatorname{dim}_{B}(\partial X) \leq d_{\sigma} \quad \text { and } \quad \operatorname{dim}_{B}\left(\partial X_{i}\right) \leq d_{\sigma} \quad(i \in \mathcal{A})
$$

with $d_{\sigma}$ as in (5.7).

Proof. In Falconer [15, Theorem 5.4] this is proved for self-affine sets rather than graph directed self-affine sets. Falconer's proof can easily be adapted to our situation (for the two-dimensional case cf. also Deliu et al. [13]). We refer the reader also to Feng et al. [19], where a similar result was established for another graph, which is more difficult to handle than the contact graph.

In some cases we can even show equality.

Proposition 5.7. Let $d \geq 2$ and let $X$ and $X_{i}(i \in \mathcal{A})$ be the atomic surfaces for a unimodular Pisot substitution $\sigma$ fulfilling the super coincidence condition. Let $\mathcal{C}_{\partial}^{\prime}$ be the essential part of $\mathcal{C}_{\partial}$. If $\mathcal{C}_{\partial}^{\prime}$ is strongly connected, and if the contracting eigenvalues of $E_{0}(\sigma)$ all have the same modulus then

$$
\operatorname{dim}_{B}(\partial X)=\operatorname{dim}_{H}(\partial X)=d_{\sigma} \quad \text { and } \quad \operatorname{dim}_{B}\left(\partial X_{i}\right)=\operatorname{dim}_{H}\left(\partial X_{i}\right)=d_{\sigma}
$$

$(i \in \mathcal{A})$ with $d_{\sigma}$ as in (5.7).

Proof. cf. Falconer [18, Corollary 3.5].

Note that Proposition 5.7 also covers the case $d=2$ and the case $d=3$ with $\lambda^{\prime}$ non-real. In order to prove $\operatorname{dim}_{B}\left(\partial X_{i}\right)=d_{\sigma}$ in a more general case we need some preparations. 
Let again $\lambda^{\prime}$ be one of the eigenvalues of $E_{0}(\sigma)$ which is smallest in modulus. We have to distinguish two cases. If $\lambda^{\prime}$ is real then there is a $j \in\{1, \ldots, s\}$ such that $\lambda^{\prime}=\lambda_{j}$. In this case define the $(d-2)$-dimensional subspace $\Pi$ by

$$
\Pi=\left\{\left(x_{2}, \ldots, x_{d}\right) \in \mathbb{R}^{d-1}: x_{j}=0\right\} .
$$

Furthermore, let $\operatorname{proj}_{\Pi}$ be the orthogonal projection from $\mathbb{R}^{d-1}$ to $\Pi$.

If $\lambda^{\prime}$ is non-real then there is a $j \in\{1, \ldots, t\}$ such that $\lambda^{\prime}=\lambda_{s+j}$. In this case define the $(d-3)$-dimensional subspace $\tilde{\Pi}$ by

$$
\tilde{\Pi}=\left\{\left(x_{2}, \ldots, x_{d}\right) \in \mathbb{R}^{d-1}: x_{s+2 j-1}=x_{s+2 j}=0\right\} .
$$

Furthermore, let $\operatorname{proj}_{\tilde{\Pi}}$ be the orthogonal projection from $\mathbb{R}^{d-1}$ to $\tilde{\Pi}$.

Lemma 5.8. Let $\mathcal{C}_{\partial}^{\prime}$ be the essential part of $\mathcal{C}_{\partial}$ and assume that $\mathcal{C}_{\partial}^{\prime}$ is strongly connected.

- If $d \geq 3$ and $\lambda^{\prime}$ is real then there is a constant $c>0$ such that

$$
\mathcal{L}_{d-2}\left(\operatorname{proj}_{\Pi}(D(V))\right)>c
$$

holds for each $V \in \mathcal{C}_{\partial}$.

- If $d \geq 4$ and $\lambda^{\prime}$ is non-real then there is a constant $c>0$ such that

$$
\mathcal{L}_{d-3}\left(\operatorname{proj}_{\tilde{\Pi}}(D(V))\right)>c
$$

holds for each $V \in \mathcal{C}_{\partial}$.

Proof. Assume that $\lambda^{\prime}$ is real. The non-real case is done in a very similar way and we omit it. Since each $T_{\mathbf{P}} X_{i}$ contains an open ball by Proposition 2.3 there is a constant $c_{1}>0$ such that

$$
\min _{i \in \mathcal{A}} \mathcal{L}_{d-2}\left(\operatorname{proj}_{\Pi}\left(T_{\mathbf{P}} \partial X_{i}\right)\right) \geq c_{1}
$$

holds. But $T_{\mathbf{P}} \partial X_{i}$ is the union of at most $\left|\mathcal{C}_{\partial}\right|$ sets of the shape $D(V)$ with $V \in \mathcal{C}_{\partial}$. Thus there is at least one element $V^{\prime} \in \mathcal{C}_{\partial}$ with

$$
\mathcal{L}_{d-2}\left(\operatorname{proj}_{\Pi}\left(D\left(V^{\prime}\right)\right)\right) \geq c_{2}
$$

for a constant $c_{2}>0$.

Case 1: $V^{\prime} \in \mathcal{C}_{\partial}^{\prime}$. Note that from each $V \in \mathcal{C}_{\partial}$ there is a walk $w$ of length $L<$ $\left|\mathcal{C}_{\partial}\right|$ leading to $V^{\prime}$. Then $D(V)$ contains a shrinked (non-rotated) copy of $D\left(V^{\prime}\right)$. By the definition of $\Pi$ we have

$$
\begin{aligned}
\mathcal{L}_{d-2}\left(\operatorname{proj}_{\Pi}(D(V))\right) & \geq \mathcal{L}_{d-2}\left(\operatorname{proj}_{\Pi}\left(\psi_{w}\left(D\left(V^{\prime}\right)\right)\right)\right) \\
& =\left|\frac{\operatorname{det} \Lambda}{\lambda^{\prime}}\right|^{L} \mathcal{L}_{d-2}\left(\operatorname{proj}_{\Pi}\left(D\left(V^{\prime}\right)\right)\right) .
\end{aligned}
$$

Thus there is a positive constant $c$ such that (5.8) holds for $V$. 
Case 2: $V^{\prime} \in \mathcal{C}_{\partial} \backslash \mathcal{C}_{\partial}^{\prime}$. In this case $D\left(V^{\prime}\right)$ is the union of finitely many shrinked (non-rotated) copies of sets of the shape $D\left(V^{\prime \prime}\right)$ with $V^{\prime \prime} \in \mathcal{C}_{\partial}^{\prime}$. Thus we reduced this case to Case 1 .

Theorem 5.9. Let $d \geq 3$ and let $X$ and $X_{i}(i \in \mathcal{A})$ be the atomic surfaces for a unimodular Pisot substitution $\sigma$ fulfilling the super coincidence condition. Let $\mathcal{C}_{\partial}^{\prime}$ be the essential part of $\mathcal{C}_{\partial}$. If $\mathcal{C}_{\partial}^{\prime}$ is strongly connected, then

$$
\operatorname{dim}_{B}(\partial X)=\operatorname{dim}_{B}\left(\partial X_{i}\right)=d_{\sigma}
$$

holds for all $i \in \mathcal{A}$ with $d_{\sigma}$ as in (5.7).

Proof. (cf. Falconer [17, Proposition 4]) The case $d=2$ as well as the case $d=3$ with $\lambda^{\prime}$ non-real is covered by Proposition 5.7 . We will confine ourselves to the case where $\lambda^{\prime}$ is real. The non-real case with $d \geq 4$ can be done with obvious modifications. We will give a lower estimate for the box counting dimension of $D(V)$ with $V \in \mathcal{C}_{\partial}$. Since for $V \notin \mathcal{C}_{\partial}^{\prime}$ the corresponding set is a finite union of sets $D\left(V^{\prime}\right)$ with $V^{\prime} \in \mathcal{C}_{\partial}^{\prime}$ we can confine ourselves to $V \in \mathcal{C}_{\partial}^{\prime}$.

Let $Y$ be as in Lemma 5.5. Then there is a $\delta>0$ satisfying

$$
\forall V \in \mathcal{C}_{\partial}^{\prime} \forall \eta \in \operatorname{proj}_{\Pi}(D(V)): \quad \mathcal{L}_{1}\left(\left\{\xi \in Y: \operatorname{proj}_{\Pi} \xi=\eta\right\}\right) \geq \delta .
$$

Fix elements $V, V^{\prime} \in \mathcal{C}_{\partial}^{\prime}$ and a walk $w \in W_{L}\left(V, V^{\prime}\right)$ for the moment. Let $\mathcal{U}$ be the $(d-1)$-dimensional unit ball. Note that $\Pi$ is the $(d-2)$-dimensional subspace of $\mathbb{R}^{d-1}$ orthogonal to the smallest axis of the ellipsoid $\psi_{w}(\mathcal{U})$. Because $\mathcal{L}_{d-2}\left(\operatorname{proj}_{\Pi}\left(D\left(V^{\prime}\right)\right)\right)>c$ holds by Lemma 5.8 , this implies that

$$
\mathcal{L}_{d-2}\left(\operatorname{proj}_{\Pi}\left(\psi_{w}\left(D\left(V^{\prime}\right)\right)\right)\right) \geq c \alpha_{1}\left(\psi_{w}\right) \cdots \alpha_{d-2}\left(\psi_{w}\right)
$$

and, for each $\eta \in \operatorname{proj}_{\Pi}\left(\psi_{w}\left(D\left(V^{\prime}\right)\right)\right)$,

$$
\mathcal{L}_{1}\left(\left\{\xi \in \psi_{w}(Y): \operatorname{proj}_{\Pi}(\xi)=\eta\right\}\right) \geq \alpha_{d-1}\left(\psi_{w}\right) \delta
$$

If $\xi \in \psi_{w}(Y)$ and $\operatorname{proj}_{\Pi}(\xi) \in \operatorname{proj}_{\Pi}\left(\psi_{w}\left(D\left(V^{\prime}\right)\right)\right)$ then $\xi$ is within distance $2 \rho \alpha_{d-1}\left(\psi_{w}\right)$ of $D(V)$ with $\rho:=\operatorname{diam}(Y)$. It follows that

$$
\begin{aligned}
\mathcal{L}_{d-1} & \left\{\xi \in \psi_{w}(Y):|\xi-\theta|<2 \rho \alpha_{d-1}\left(\psi_{w}\right) \text { for some } \theta \in D(V)\right\} \\
& \geq \mathcal{L}_{d-2}\left(\operatorname{proj}_{\Pi}\left(\psi_{w}\left(D\left(V^{\prime}\right)\right)\right)\right) \alpha_{d-1}\left(\psi_{w}\right) \delta \\
& \geq c \delta \alpha_{1}\left(\psi_{w}\right) \cdots \alpha_{d-1}\left(\psi_{w}\right)
\end{aligned}
$$

By Lemma 5.5, $\bigcup_{w \in W_{L}} \psi_{w}(Y)$ is a union of sets which covers each point in $\mathbb{R}^{d-1}$ for at most $\nu$ times. Obviously, the same assertion is true for $\bigcup_{w \in W_{L}(V)} \psi_{w}(Y)$. Note that $h:=\alpha_{d-1}\left(\psi_{w}\right)$ is independent of $w \in W_{L}(V)$. 
We have

$$
\begin{aligned}
\mathcal{L}_{d-1}\{\xi & \left.\in \mathbb{R}^{d-1}:|\xi-\theta| \leq 2 \rho h \text { for some } \theta \in D(V)\right\} \\
& \geq \mathcal{L}_{d-1}\left(\bigcup_{w \in W_{L}(V)}\left\{\xi \in \psi_{w}(Y):|\xi-\theta|<2 \rho h \text { for some } \theta \in D(V)\right\}\right) \\
& \geq \nu^{-1} \sum_{w \in W_{L}(V)} \mathcal{L}_{d-1}\left\{\xi \in \psi_{w}(Y):|\xi-\theta|<2 \rho h \text { for some } \theta \in D(V)\right\} \\
& \geq \frac{c \delta}{\nu} \sum_{w \in W_{L}(V)} \alpha_{1}\left(\psi_{w}\right) \cdots \alpha_{d-1}\left(\psi_{w}\right) \\
& =\frac{c \delta}{\nu} \sum_{w \in W_{L}(V)} \alpha_{1}\left(\psi_{w}\right) \cdots \alpha_{d-1}\left(\psi_{w}\right)^{\kappa-(d-1)+1} h^{(d-1)-\kappa} \\
& =\frac{c \delta h^{(d-1)-\kappa}}{\nu} \sum_{w \in W_{L}(V)} \phi^{\kappa}\left(\psi_{w}\right)
\end{aligned}
$$

for $d-2<\kappa \leq d-1$. If $\kappa<d_{\sigma}$ then (5.6) implies that $\sum_{w \in W_{L}(V)} \phi^{\kappa}\left(\psi_{w}\right) \geq$ $M$ for some constant $M$ not depending on $h$. Thus

$$
\mathcal{L}_{d-1}\left\{\xi \in \mathbb{R}^{d-1}:|\xi-\theta| \leq 2 \rho h \text { for some } \theta \in D(V)\right\} \geq c_{1} h^{(d-1)-\kappa} .
$$

Using [16, Proposition 3.2] this implies that $\operatorname{dim}_{B}(D(V)) \geq d_{\sigma}$ holds for each $V \in \mathcal{C}_{\partial}^{\prime}$. Together with Proposition 5.6 this implies the theorem.

\section{An example: A class of cubic unimodular Pisot substitutions}

The remaining part of the paper is devoted to unimodular Pisot substitutions of the shape

$$
\sigma(1)=\underbrace{1 \ldots 1}_{b \text { times }} 2, \quad \sigma(2)=\underbrace{1 \ldots 1}_{a \text { times }} 3, \quad \sigma(3)=1
$$

with $1 \leq a \leq b$. Note that all these substitutions fulfill the super coincidence condition. In particular in Solomyak [41] it is shown that they have pure discrete spectrum and Barge and Kwapisz [8] prove that pure discrete spectrum is equivalent to the super coincidence condition.

The substitutions in (6.1) are special instances of the substitutions (2.6) that are related to $\beta$-expansions of real numbers. The choice $a=b=1$ yields the Tribonacci substitution. It turns out that most of these substitutions have essentially one and the same contact graph $\mathcal{C}$.

Let $\sigma$ be as in (6.1). Then we easily see that its adjacency matrix $E_{0}(\sigma)$ is given by

$$
E_{0}(\sigma)=\left(\begin{array}{lll}
b & a & 1 \\
1 & 0 & 0 \\
0 & 1 & 0
\end{array}\right)
$$


6.1. The eigenvector of the dominant eigenvalue of $E_{0}(\sigma)$. Before we can construct the contact graphs of this class of substitutions we need the following result on the positive left eigenvector $v$ of $E_{0}(\sigma)$ corresponding to its dominant eigenvalue.

Proposition 6.1. Let $\sigma$ be as in (6.1) and let $v=v_{1}=\left(v_{11}, v_{12}, v_{13}\right)$ be the left eigenvector corresponding to the dominant eigenvalue of $E_{0}(\sigma)$ having $v_{13}=1$. Then $v_{1 j}(1 \leq j \leq 3)$ is positive and we have

$$
v_{11}>v_{12}>v_{13}=1 .
$$

Proof. See [14, Section 8, p. 223], where $v_{11}, v_{12}$ and $v_{13}$ have been calculated explicitly.

In Figure 8 two examples of atomic surfaces corresponding to substitutions of the class (6.1) are given.
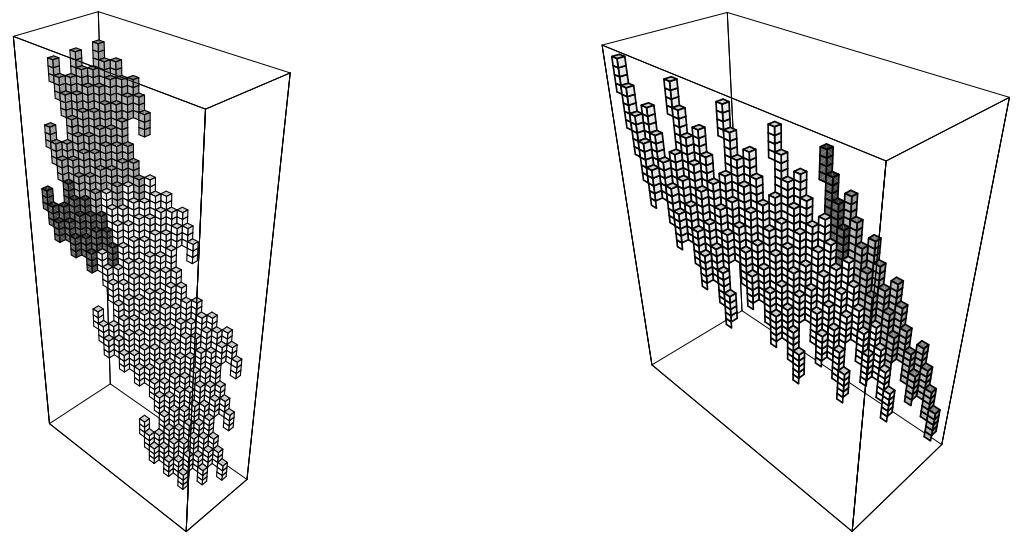

FiguRE 8 . The left atomic surface corresponds to the choice $a=b=2$, the right one to $a=3, b=4$.

6.2. Calculation of contact matrices. Now we want to determine the contact graph of the substitutions in (6.1). This could be done directly by using Definition 3.6. However, in order to shorten the procedure, with help of a Mathematica ${ }^{\circledR}$ program we calculated the contact graph for several choices of $a$ and $b$. This led us to conjecture that in the general case the contact graph has set of states $M(a, b)$, where (all elements are written in 
canonical order)

$$
M(a, b):= \begin{cases}M \cup\left\{\left(\left[0,1^{*}\right],\left[(1,-1,1), 1^{*}\right]\right),\right. & \text { if } b>a>1, \\ \left.\left(\left[0,1^{*}\right],\left[(1,0,-1), 1^{*}\right]\right)\right\} & \\ M \cup\left\{\left(\left[0,1^{*}\right],\left[(1,-1,1), 1^{*}\right]\right)\right\} & \text { if } b=a>1, \\ M \cup\left\{\left(\left[0,1^{*}\right],\left[(1,0,-1), 1^{*}\right]\right)\right\} & \text { if } b>a=1, \\ M & \text { if } b=a=1 .\end{cases}
$$

with

$$
\begin{aligned}
M:= & \left(\left[0,1^{*}\right],\left[0,1^{*}\right]\right),\left(\left[0,1^{*}\right],\left[0,2^{*}\right]\right),\left(\left[0,1^{*}\right],\left[0,3^{*}\right]\right), \\
& \left(\left[0,1^{*}\right],\left[(0,0,1), 1^{*}\right]\right),\left(\left[0,1^{*}\right],\left[(0,0,1), 2^{*}\right]\right), \\
& \left(\left[0,1^{*}\right],\left[(0,1,-1), 1^{*}\right]\right),\left(\left[0,1^{*}\right],\left[(0,1,-1), 2^{*}\right]\right), \\
& \left(\left[0,1^{*}\right],\left[(0,1,0), 1^{*}\right]\right),\left(\left[0,2^{*}\right],\left[0,3^{*}\right]\right),\left(\left[0,2^{*}\right],\left[(0,0,1), 2^{*}\right]\right), \\
& \left(\left[0,2^{*}\right],\left[(1,-1,0), 1^{*}\right]\right),\left(\left[0,2^{*}\right],\left[(1,-1,1), 1^{*}\right]\right), \\
& \left(\left[0,2^{*}\right],\left[(1,0,-1), 1^{*}\right]\right),\left(\left[0,3^{*}\right],\left[0,3^{*}\right]\right), \\
& \left.\left(\left[0,3^{*}\right],\left[(0,1,-1), 2^{*}\right]\right),\left(\left[0,3^{*}\right],\left[(1,0,-1), 1^{*}\right]\right)\right\} .
\end{aligned}
$$

Using Definition 3.6 we can calculate the graph $G(M(a, b))$ by a lengthy but easy computation. Note that for the calculation of the box counting dimension we only need subgraphs of the graph $\mathcal{C}_{\partial}$ defined in Definition 4.5. Indeed, the quantity $d_{\sigma}$ in (5.7) which occurs in the dimension formulas of Proposition 5.7 and Theorem 5.9 depends only on $E_{0}(\sigma)$ and $\mathcal{C}_{\partial}$. Thus the states $\left(\left[0, i^{*}\right],\left[0, i^{*}\right]\right)$ are of no importance in what follows and we will leave them away.

Lemma 6.2. Let $M^{\prime}(a, b)=M(a, b) \backslash\left\{\left(\left[0, i^{*}\right],\left[0, i^{*}\right]\right): 1 \leq i \leq 3\right\}$.

- If $b>a>1$ then $G\left(M^{\prime}(a, b)\right)$ is depicted in Figure $9^{1}$.

- If $b=a>1$ then $G\left(M^{\prime}(a, b)\right)$ is depicted in Figure $10^{1}$.

- If $b>a=1$ then $G\left(M^{\prime}(a, b)\right)$ emerges from the graph in Figure 9 by deleting the state $\left(\left[0,1^{*}\right],[(1,-1,1), 1]\right)$ and all the broken-line edges, and by adding the edge

$$
\left(\left[0,2^{*}\right],\left[(0,0,1), 2^{*}\right]\right) \stackrel{1}{\rightarrow}\left(\left[0,1^{*}\right],\left[(0,1,-1), 1^{*}\right]\right) .
$$

\footnotetext{
${ }^{1}$ We are only interested in the number of edges leading from one state to another. Each label $\ell$ in the figure represents $\ell$ edges.
} 


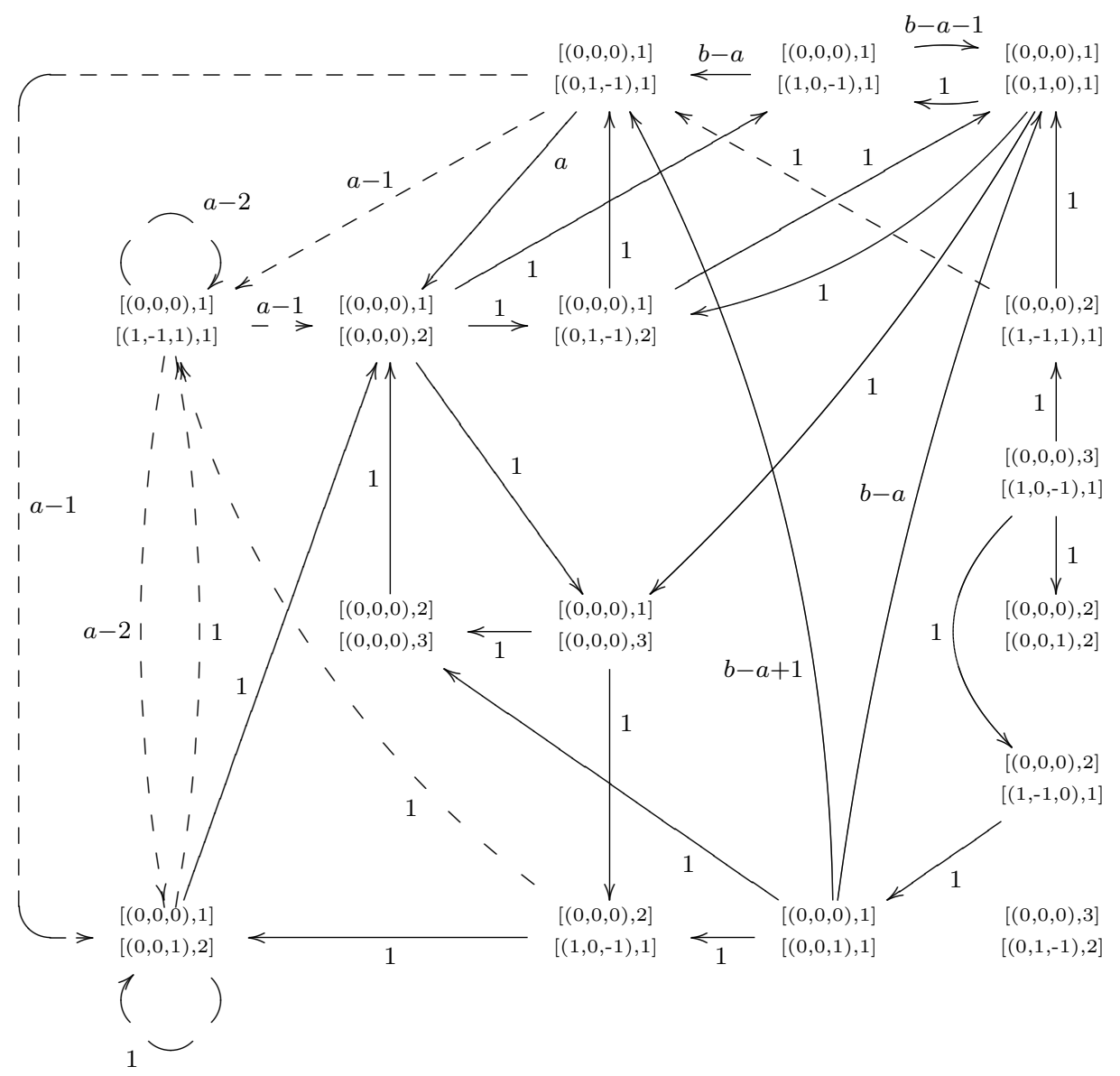

Figure 9. A picture of the graph $G\left(M^{\prime}(a, b)\right)$ for $b>a>1$. Each edge labelled by $\ell$ in the picture represents $\ell$ edges in $G\left(M^{\prime}(a, b)\right)$. If $\ell \leq 0$ then the corresponding edge does not exist.

- If $b=a=1$ then $G\left(M^{\prime}(a, b)\right)$ emerges from the graph in Figure 10 by deleting the state $\left(\left[0,1^{*}\right],\left[(1,-1,1), 1^{*}\right]\right)$ and all the broken-line edges, and by adding the edges in (6.3).

Having deduced the shape of these graphs we can easily prove the following result.

Lemma 6.3. Let $\sigma$ be a substitution of the shape (6.1). Then $R_{0} \subseteq M(a, b) \subseteq$ $R$.

Proof. The first inclusion can be checked directly by observing that $M(a, b)$ contains all unit tips that pair faces with unit tips of the form $\left(\left[0, i^{*}\right],\left[0, j^{*}\right]\right)$. 


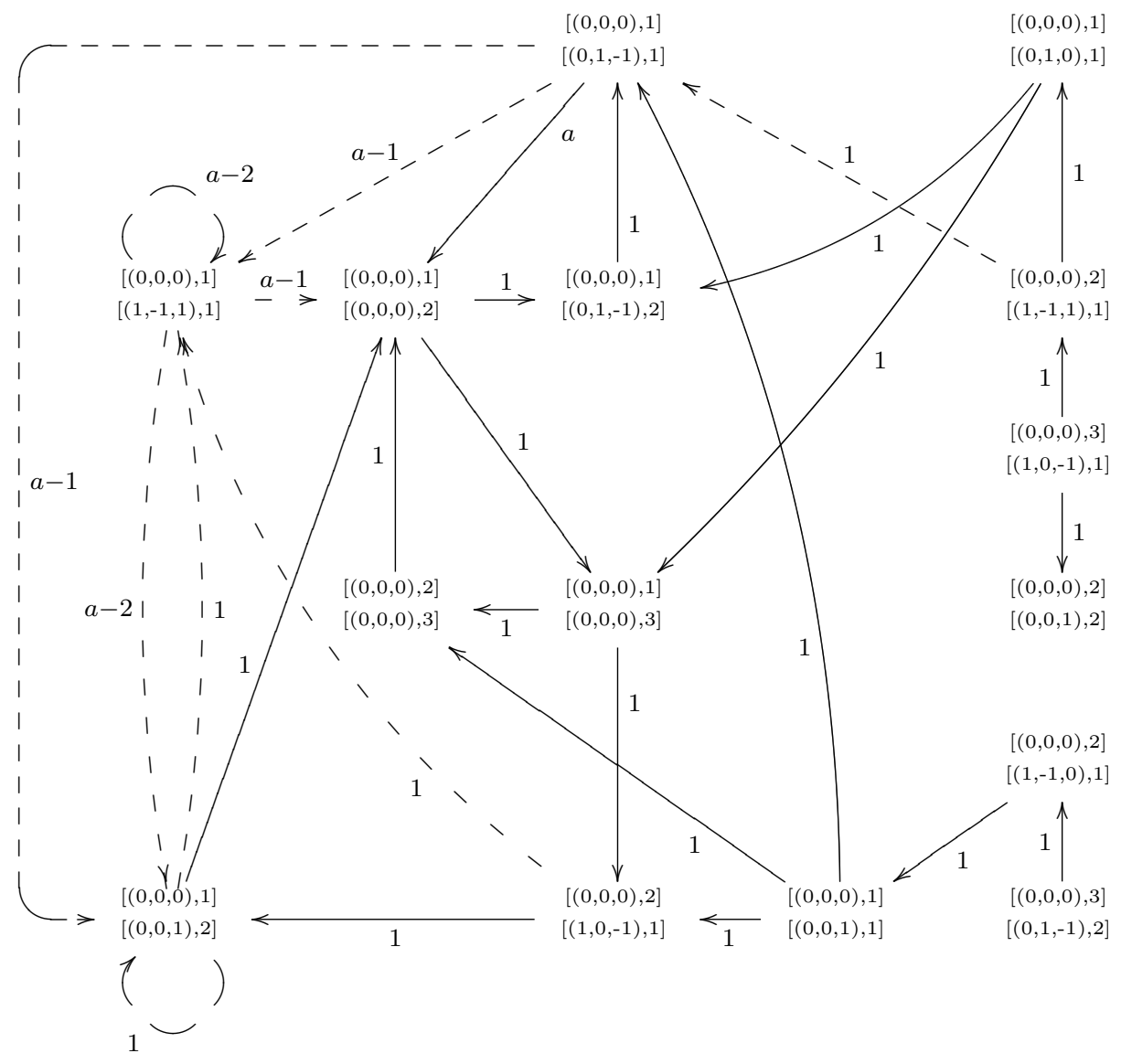

FiguRE 10. A picture of the graph $G\left(M^{\prime}(a, b)\right)$ for $b=a>1$.

The second inclusion is a consequence of Lemma 3.8. For all states $v$ of $G(M(a, b))$ it is easily seen that $v$ is either contained in $R_{0}$ or there leads a walk from $v$ to the state $\left(\left[0,1^{*}\right],\left[0,2^{*}\right]\right) \in R_{0}$.

Our goal is to prove the following theorem.

Theorem 6.4. Let $\sigma$ be a cubic unimodular Pisot substitution of the shape (6.1) with $b \geq a \geq 1$. Then the contact graph $\mathcal{C}$ of $\sigma$ is given by

$$
\mathcal{C}=G(M(a, b))
$$

where $M(a, b)$ is defined as in (6.2). Thus the number of states of $\mathcal{C}$ is either 17 or 18 or 19.

Remark 6.5. The shape of the graph $\mathcal{C}_{\partial}$ can easily be derived from Figure 9 and Figure 10 via Lemma 6.2. 
6.3. Proof of Theorem 6.4. We already proved that $R_{0} \subseteq M(a, b) \subseteq R$. Thus in view of Corollary 3.5 we have to show that $\Psi(M(a, b))=M(a, b)$ in order to prove Theorem 6.4. This will be done in the following lemma.

Lemma 6.6. Let $\sigma$ be as in (6.1) with $1 \leq a \leq b$. Then $M(a, b)=$ $\Psi(M(a, b))$ and thus $G(M(a, b))=\mathcal{C}$ is the contact graph of $\sigma$.

Proof. We will show the result only for the case $b>a>1$. The three other cases follow by arguing along the same lines with minor modifications.

We easily see that

$$
\begin{aligned}
& V_{1}:=E_{1}(\sigma)[0,1]=\{[0,2],[(0,-1,0), 1], \ldots,[(1-b,-1,0), 1]\}, \\
& V_{2}:=E_{1}(\sigma)[0,2]=\{[0,3],[(0,0,-1), 1], \ldots,[(1-a, 0,-1), 1]\}, \\
& V_{3}:=E_{1}(\sigma)[0,3]=\{[0,1]\}
\end{aligned}
$$

Also the following list of identities will be useful during the proof.

$$
\begin{aligned}
E_{1}(\sigma)[(0,0,1), 1]=\{[ & (1,0,0), 2],[(1,-1,0), 1], \ldots, \\
& {[(2-b,-1,0), 1]\}, } \\
E_{1}(\sigma)[(0,0,1), 2]=\{[(1,0,0), 3],[(1,0,-1), 1], \ldots, & {[(2-a, 0,-1), 1]\}, } \\
E_{1}(\sigma)[(0,1,-1), 1]=\{[(a-1,0,1), 2],[(a-1,-1,1), 1], \ldots, & {[(a-b,-1,1), 1]\}, } \\
E_{1}(\sigma)[(0,1,-1), 2]=\{[(a-1,0,1), 3],[(a-1,0,0), 1], \ldots, & {[(0,0,0), 1]\} } \\
E_{1}(\sigma)[(0,1,0), 1]=\{[(a, 0,1), 2],[(a,-1,1), 1], \ldots, & {[(a+1-b,-1,1), 1]\}, } \\
E_{1}(\sigma)[(1,-1,1), 1]=\{[(b-a+1,1,-1), 2],[(b-a+1,0,-1), 1], \ldots, & {[(2-a, 0,-1), 1]\}, } \\
E_{1}(\sigma)[(1,0,-1), 1]=\{[(b-1,1,0), 2],[(b-1,0,0), 1], \ldots, & {[(0,0,0), 1]\}, } \\
E_{1}(\sigma)[(1,-1,0), 1]=\{[(b-a, 1,-1), 2],[(b-a, 0,-1), 1], \ldots, & {[(1-a, 0,-1), 1]\} . }
\end{aligned}
$$

Let $E$ be the set of all unit tips $\left[z, l^{*}\right]$ for which there exists an $i \in \mathcal{A}$ with $\left(\left[0, k^{*}\right],\left[z, l^{*}\right]\right) \in M(a, b)$. We will have to form $E_{1}(\sigma)[z, l]$ of all $\left[z, l^{*}\right] \in E$. 
Using (6.4) and (6.5) we get

$$
\begin{aligned}
\bigcup_{\left[z, l^{*}\right] \in E} E_{1}(\sigma)[z, l] & :=\{[(m, 0,-1), 1]: 1-a \leq m \leq b-a+1\} \\
& \cup\{[(m, 1,-1), 2]: b-a \leq m \leq b-a+1\} \\
\cup & \{[(b-1,1,0), 2]\} \\
\cup & \{[(m,-1,1), 1]: a-b \leq m \leq a\} \\
\cup & \{[(m, 0,0), j]: 0 \leq m \leq b-1 \text { for } j=1 \text { and } \\
& 0 \leq m \leq 1 \text { for } j=2,3\} \\
\cup & \{[(m,-1,0), 1]: 1-b \leq m \leq 1\} \\
\cup\{[(m, 0,1), j]: a-1 \leq m \leq a \text { for } j=2 \text { and } & \\
& m=a-1 \text { for } j=3\} .
\end{aligned}
$$

For abbreviation we call the unions on the right hand side $U_{1}, \ldots, U_{7}$.

In order to prove the lemma we will use directly the definition of $\Psi$ : First we have to form all elements of the shape $\varphi\left(\left[x, i^{*}\right],\left[y, j^{*}\right]\right)$ with $[y, j] \in$ $U_{1} \cup \ldots \cup U_{7}$ and $[x, i] \in V_{1} \cup V_{2} \cup V_{3}$. If there exists $\left(\left[0, k^{*}\right],\left[z, l^{*}\right]\right) \in M(a, b)$ such that

$$
[x, i] \in E_{1}(\sigma)[0, k] \quad \text { and } \quad[y, j] \in E_{1}(\sigma)[z, l]
$$

then we have show that $\varphi\left(\left[x, i^{*}\right],\left[y, j^{*}\right]\right) \in M(a, b)$ provided that the second coordinate of $\varphi$ is a unit tip (the first one is always a unit tip). By the definition of $\Psi$ this will imply $\Psi(M(a, b)) \subset M(a, b)$. Since the other direction is trivial this finishes the proof.

To avoid trivial repetitions, we will give the proof only for $[y, j] \in U_{1}$. Thus we have to consider all elements $\varphi\left(\left[x, i^{*}\right],\left[y, j^{*}\right]\right)$ where $[x, i] \in V_{1} \cup$ $V_{2} \cup V_{3}$ and $[y, j]=[(m, 0,-1), 1]$ with $1-a \leq m \leq b-a+1$.

First we assume that $[x, i]=[0, i]$. Thus Proposition 6.1 together with the definition of $\varphi$ yields that

$$
\varphi\left(\left[0, i^{*}\right],\left[(m, 0,-1), 1^{*}\right]\right)= \begin{cases}\left(\left[0, i^{*}\right],\left[(m, 0,-1), 1^{*}\right]\right) & m>0 \\ \left(\left[0,1^{*}\right],\left[(-m, 0,1), i^{*}\right]\right) & m \leq 0\end{cases}
$$

Using Proposition 6.1 and the definition of "unit tip" from Subsection 2.5 we see that this contains the pairs of unit tips

$$
\begin{aligned}
\left(\left[0, i^{*}\right],\left[(1,0,-1), 1^{*}\right]\right) & (1 \leq i \leq 3), \\
\left(\left[0,1^{*}\right],\left[(0,0,1), i^{*}\right]\right) & (1 \leq i \leq 2) .
\end{aligned}
$$

Since each of these pairs is contained in $M(a, b)$ we are done. 
Now assume that $[x, i]=[(q,-1,0), 1]$ with $q \leq 0$. Then Proposition 6.1 implies that

$$
\varphi\left(\left[(q,-1,0), 1^{*}\right],\left[(m, 0,-1), 1^{*}\right]\right)= \begin{cases}\left(\left[0,1^{*}\right],\left[(m-q, 1,-1), 1^{*}\right]\right) & m \geq q \\ \left(\left[0,1^{*}\right],\left[(q-m,-1,1), 1^{*}\right]\right) & m<q .\end{cases}
$$

The same proposition yields that the only pairs of unit tips occurring here are

$$
\left(\left[0,1^{*}\right],\left[(0,1,-1), 1^{*}\right]\right) \text { and }\left(\left[0,1^{*}\right],\left[(1,-1,1), 1^{*}\right]\right)
$$

Both of them are contained in $M(a, b)$.

Next assume that $[x, i]=[(q, 0,-1), 1]$ with $q \leq 0$. Then

$$
\varphi\left(\left[(q, 0,-1), 1^{*}\right],\left[(m, 0,-1), 1^{*}\right]\right)= \begin{cases}\left(\left[0,1^{*}\right],\left[(m-q, 0,0), 1^{*}\right]\right) & m \geq q \\ \left(\left[0,1^{*}\right],\left[(q-m, 0,0), 1^{*}\right]\right) & m<q\end{cases}
$$

The only pair of unit tips contained here is $\left(\left[0,1^{*}\right],\left[0,1^{*}\right]\right)$ which is contained in $M(a, b)$. Since we covered all $[x, i] \in V_{1} \cup V_{2} \cup V_{3}$ the case $[y, j] \in U_{1}$ is settled.

Doing the same for the other $[y, j] \in U_{\ell}(\ell \in\{2,3,4,5,6,7\})$, we see that there does not occur any new element in $\Psi(M(a, b))$. Thus $\Psi(M(a, b))$ only contains elements of $M(a, b)$ and we are done.

\subsection{Calculation of the Hausdorff and box counting dimension.} In the present section we want to establish explicit formulas for the box counting dimension of the tiles related to the substitutions in (6.1). First of all we note that the contact graph associated to each of these substitutions satisfies the conditions of Theorem 5.9. In order to apply this theorem we may work with the reduced graph $\mathcal{C}_{\partial}^{\prime}$ rather than $\mathcal{C}_{\partial}$. According to the four different classes of contact graphs we have to distinguish four cases.

First we mention that the characteristic polynomial of $E_{0}(\sigma)$ is

$$
x^{3}-b x^{2}-a x-1
$$

Thus the two roots contained in the unit circle are different and real if and only if

$$
D:=\frac{1}{108}\left(27-4 a^{3}+18 a b-a^{2} b^{2}+4 b^{3}\right)<0 .
$$

Thus the occurring fractals are graph directed self-similar if and only if $D \geq 0$. 
First consider the case $b>a>1$. It is easy to see that the adjacency matrix of $\mathcal{C}_{\partial}^{\prime}$ in this case is given by

$$
M_{1}:=\left(\begin{array}{cccccccccc}
0 & 0 & 0 & a-1 & a & 0 & 0 & 0 & a-1 & 0 \\
b-a & 0 & b-a-1 & 0 & 0 & 0 & 0 & 0 & 0 & 0 \\
0 & 1 & 0 & 0 & 0 & 1 & 0 & 1 & 0 & 0 \\
0 & 0 & 0 & a-2 & a-1 & 0 & 0 & 0 & a-2 & 0 \\
0 & 1 & 0 & 0 & 0 & 1 & 0 & 1 & 0 & 0 \\
1 & 0 & 1 & 0 & 0 & 0 & 0 & 0 & 0 & 0 \\
0 & 0 & 0 & 0 & 1 & 0 & 0 & 0 & 0 & 0 \\
0 & 0 & 0 & 0 & 0 & 0 & 1 & 0 & 0 & 1 \\
0 & 0 & 0 & 1 & 1 & 0 & 0 & 0 & 1 & 0 \\
0 & 0 & 0 & 1 & 0 & 0 & 0 & 0 & 1 & 0
\end{array}\right) .
$$

We can read off from Figure 9 that $\mathcal{C}_{\partial}^{\prime}$ is strongly connected. Furthermore, from the same figure we see that the greatest common divisor of the length of its cycles is equal to 1 . This implies that $\mathcal{C}_{\partial}^{\prime}$ is primitive and thus it has a uniquely defined positive largest eigenvalue $\mu$, which is the largest root of its characteristic polynomial. The characteristic polynomial splits up in several irreducible factors. The one contributing the largest root $\mu$ is easily seen to be

$$
p_{a, b}(x)=x^{4}+(1-a) x^{3}+(a-b) x^{2}-(b+1) x-1 .
$$

For the three other cases we also find the largest root of the characteristic polynomial easily. In the case $b=a>1$ it is the largest root of $p_{b, b}(x)$, for $b>a=1$ it is the largest root of $p_{b, 1}(x)$ and for the Tribonacci substitution corresponding to the choice $b=a=1$ it is the largest root of $p_{1,1}(x)$.

Thus Proposition 5.7 and Theorem 5.9 yield the following result.

Theorem 6.7. Let $X, X_{1}, X_{2}$ and $X_{3}$ be the atomic surfaces associated to the substitution

$$
\sigma(1)=\underbrace{1 \ldots 1}_{\text {btimes }} 2, \quad \sigma(2)=\underbrace{1 \ldots 1}_{\text {atimes }} 3, \quad \sigma(3)=1
$$

with $b \geq a \geq 1$. Let $\mu$ be the positive largest root (in modulus) of the polynomial

$$
x^{4}+(1-a) x^{3}+(a-b) x^{2}-(b+1) x-1,
$$

$\lambda^{\prime}$ shall be one of the the smallest (in modulus) eigenvalues of $E_{0}(\sigma)$. Then the box counting dimension of $\partial X_{i}(1 \leq i \leq 3)$ and $\partial X$ is given by

$$
\operatorname{dim}_{B}(\partial X)=\operatorname{dim}_{B}\left(\partial X_{i}\right)=2+\frac{\log \lambda-\log \mu}{\log \left|\lambda^{\prime}\right|} \quad(i \in\{1,2,3\}) .
$$

If $D \geq 0$ then we also have

$\operatorname{dim}_{H}(\partial X)=\operatorname{dim}_{B}(\partial X) \quad$ and $\quad \operatorname{dim}_{H}\left(\partial X_{i}\right)=\operatorname{dim}_{B}\left(\partial X_{i}\right) \quad(i \in\{1,2,3\})$. 


\section{Acknowledgement}

I am indebted to Valerie Berthé and Wolfgang Steiner for valuable discussions and suggestions. I also want to thank the anonymous referee. Her or his detailed suggestions improved the paper considerably.

\section{References}

[1] Akiyama, S. Self affine tilings and Pisot numeration systems. In Number Theory and its Applications (1999), K. Győry and S. Kanemitsu, Eds., Kluwer, pp. 1-17.

[2] Akiyama, S. On the boundary of self affine tilings generated by Pisot numbers. J. Math. Soc. Japan 54, 2 (2002), 283-308.

[3] Akiyama, S., Sadahiro, T. A self-similar tiling generated by the minimal Pisot number. Acta Math. Info. Univ. Ostraviensis 6 (1998), 9-26.

[4] Arnoux, P., Berthé, V., Ei, H., Ito, S. Tilings, quasicrystals, discrete planes, generalized substitutions, and multidimensional continued fractions. In Discrete models: combinatorics, computation, and geometry (Paris, 2001), Discrete Math. Theor. Comput. Sci. Proc., AA. Maison Inform. Math. Discrèt. (MIMD), Paris, 2001, pp. 059-078 (electronic).

[5] Arnoux, P., Berthé, V. Siegel, A. Two-dimensional iterated morphisms and discrete planes. Theoret. Comput. Sci. 319, 1-3 (2004), 145-176.

[6] Arnoux, P. Ito, S. Pisot substitutions and Rauzy fractals. Bull. Belg. Math. Soc. Simon Stevin 8, 2 (2001), 181-207. Journées Montoises d'Informatique Théorique (Marne-la-Vallée, 2000).

[7] Barge, M. Diamond, B. Coincidence for substitutions of Pisot type. Bull. Soc. Math. France 130, 4 (2002), 619-626.

[8] Barge, M. Kwapisz, J. Geometric theory of unimodular Pisot substitutions. Preprint.

[9] Berthé, V. Siegel, A. Purely periodic beta-expansions in the Pisot non-unit case. Preprint.

[10] Berthé, V. Siegel, A. Tilings associated with beta-numeration and substitutions. Integers 5 (2005), no. 3, A2, (electronic).

[11] Canterini, V. Siegel, A. Automate des préfixes-suffixes associé à une substitution primitive. J. Théor. Nombres Bordeaux 13, 2 (2001), 353-369.

[12] Canterini, V. Siegel, A. Geometric representation of substitutions of Pisot type. Trans. Amer. Math. Soc. 353, 12 (2001), 5121-5144 (electronic).

[13] Deliu, A., Geronimo, J., Shonkwiler, R. Hardin, D. Dimension associated with recurrent self similar sets. Math. Proc. Camb. Philos. Soc. 110 (1991), 327-336.

[14] Dumont, J. M. Thomas, A. Digital sum moments and substitutions. Acta Arith. 64 (1993), $205-225$.

[15] Falconer, K. J. The Hausdorff dimension of self-affine fractals. Math. Proc. Camb. Phil. Soc. 103 (1988), 339-350.

[16] Falconer, K. J. Fractal Geometry. John Wiley and Sons, Chichester, 1990.

[17] Falconer, K. J. The dimension of self-affine fractals II. Math. Proc. Camb. Phil. Soc. 111 (1992), 169-179.

[18] Falconer, K. J. Techniques in Fractal Geometry. John Wiley and Sons, Chichester, New York, Weinheim, Brisbane, Singapore, Toronto, 1997.

[19] Feng, D.-J., Furukado, M., Ito, S. Wu, J. Pisot substitutions and the Hausdorff dimension of boundaries of atomic surfaces. Tsukuba J. Math. 30 (2006), 195-224.

[20] FogG, N. P. Substitutions in dynamics, arithmetics and combinatorics. Vol. 1794 of Lecture Notes in Mathematics. Springer-Verlag, Berlin, 2002. Edited by V. Berthé, S. Ferenczi, C. Mauduit and A. Siegel.

[21] Frougny, C. Solomyak, B. Finite beta-expansions. Ergodic Theory Dynam. Systems 12, 4 (1992), 713-723.

[22] Gröchenig, K. HaAs, A. Self-similar lattice tilings. J. Fourier Anal. Appl. 1 (1994), 131170.

[23] Holton, C. Zamboni, L. Q. Geometric realizations of substitutions. Bull. Soc. Math. France 126, 2 (1998), 149-179. 
[24] Ito, S. Kimura, M. On Rauzy fractal. Japan J. Indust. Appl. Math. 8, 3 (1991), 461-486.

[25] Iто, S. RAO, H. Atomic surfaces, Tilings and coincidence I. Irreducible case. Israel J. Math., to appear.

[26] Ito, S. SAno, Y. Substitutions, atomic surfaces, and periodic beta expansions. In Analytic number theory (Beijing/Kyoto, 1999), vol. 6 of Dev. Math. Kluwer Acad. Publ., Dordrecht, 2002, pp. 183-194.

[27] Lind, D. Marcus, B. An introduction to symbolic dynamics and coding. Cambridge University Press, Cambridge, 1995.

[28] Mauldin, R. D. Williams, S. C. Hausdorff dimension in graph directed constructions. Trans. Amer. Math. Soc. 309 (1988), 811-829.

[29] Messaoudi, A. Propriétés arithmétiques et dynamiques du fractal de Rauzy. J. Théor. Nombres Bordeaux 10, 1 (1998), 135-162.

[30] Messaoudi, A. Frontière du fractal de Rauzy et système de numération complexe. Acta Arith. 95, 3 (2000), 195-224.

[31] Mossé, B. Recognizability of substitutions and complexity of automatic sequences. Bull. Soc. Math. Fr. 124, 2 (1996), 329-346.

[32] PARry, W. On the $\beta$-expansions of real numbers. Acta Math. Acad. Sci. Hungar. 11 (1960), 401-416.

[33] Queffélec, M. Substitution dynamical systems-spectral analysis. Vol. 1294 of Lecture Notes in Mathematics. Springer-Verlag, Berlin, 1987.

[34] RAuZy, G. Nombres algébriques et substitutions. Bull. Soc. Math. France 110, 2 (1982), 147-178.

[35] Sano, Y., Arnoux, P. Ito, S. Higher dimensional extensions of substitutions and their dual maps. J. Anal. Math. 83 (2001), 183-206.

[36] Scheicher, K. Thuswaldner, J. M. Canonical number systems, counting automata and fractals. Math. Proc. Cambridge Philos. Soc. 133, 1 (2002), 163-182.

[37] Scheicher, K. Thuswaldner, J. M. Neighbours of self-affine tiles in lattice tilings. In Proceedings of the Conference "Fractals in Graz" (2002), P. Grabner and W. Woess, Eds., pp. 241-262.

[38] Siegel, A. Représentation des systèmes dynamiques substitutifs non unimodulaires. Ergodic Theory Dynam. Systems 23, 4 (2003), 1247-1273.

[39] Siegel, A. Pure discrete spectrum dynamical system and periodic tiling associated with a substitution. Ann. Inst. Fourier (Grenoble) 54, 2 (2004), 341-381.

[40] Sirvent, V. F. WAng, Y. Self-affine tiling via substitution dynamical systems and Rauzy fractals. Pacific J. Math. 206, 2 (2002), 465-485.

[41] Solomyak, B. Substitutions, adic transformations, and beta-expansions. In Symbolic dynamics and its applications (New Haven, CT, 1991). Vol. 135 of Contemp. Math. Amer. Math. Soc., Providence, RI, 1992, pp. 361-372.

[42] Thurston, W. Groups, tilings and finite state automata. AMS Colloquium Lecture Notes, 1989.

[43] Vince, A. Digit tiling of euclidean space. In Directions in Mathematical Quasicrystals (Providence, RI, 2000), Amer. Math. Soc., pp. 329-370.

Jörg M. THuswaLdner

Institut für Mathematik und Angewandte Geometrie

Abteilung für Mathematik und Statistik

Montanuniversität Leoben

Franz-Josef-Strasse 18

A-8700 LEOBEN, AUSTRIA 